\title{
Convergence of best-response dynamics in extensive-form games
}

\author{
Zibo $\mathrm{Xu}^{1}$ \\ Department of Economics, Stockholm School of Economics \\ SSE/EFI Working Paper Series in Economics and Finance \\ No 745 \\ June 2013
}

\begin{abstract}
We prove that in all finite generic extensive-form games of perfect information, a continuous-time best-response dynamic always converges to a Nash equilibrium component. We show the robustness of convergence by an approximate best-response dynamic: for any initial state and allowed approximate best-response dynamic, the state is close to the set of Nash equilibria most of the time. We also prove that in a perfect-information game where each player can move at only one node, all interior approximate best-response dynamics converge to the backward-induction equilibrium, which is hence the socially stable strategy in the game.
\end{abstract}

\section{JEL classification: C73, D83.}

Keywords: Convergence to Nash equilibrium, games in extensive form, games of perfect information, Nash equilibrium components, best-response dynamics, fictitious play, socially stable strategy.

\section{Introduction}

In uncoupled dynamics, a player's strategy is independent of the payoff functions of the other players, but it need not be independent of the player's own payoff function or the other players' strategies. The rules of behavior in

\footnotetext{
${ }^{1}$ The author is grateful to Sergiu Hart and Jörgen Weibull for many suggestions and discussions. The author also wishes to thank Carlos Alós-Ferrer, Itai Arieli, Larry Samuelson, Bill Sandholm, Tomas Sjöström and Eyal Winter for their comments. The author would like to acknowledge financial support from the Knut and Alice Wallenberg Foundation.
} 
such situations are called adaptive heuristics (Hart, 2005), as they are simple, natural, and (possibly naively) payoff-improving. Some common adaptive heuristics are best-reply dynamics, better-reply dynamics, and fictitious plays.

Uncoupled dynamics are often applied to justify solution concepts in games. The question that is asked is, what dynamic process leads to what equilibrium, and in what kind of games. To answer this question, Hofbauer and Sandholm (2002) and Hart and Mas-Colell (2003) applied uncoupled dynamics to zero-sum games, potential games, dominance-solvable games, and more. They found that for these special classes of games, some uncoupled dynamics can guarantee convergence to the set of Nash equilibria. Hart and Mas-Colell (2005) later proved the general negative result that there exists no uncoupled dynamics that guarantees Nash convergence in all games.

In the present paper, we confine our attention to best-response dynamics. A best-response dynamic is often viewed as a basic and natural process when we studying long-run behavior in a repeated game. To be specific, in a game with large populations of boundedly rational individuals and limited information about the dynamic process, each player may refer to best replies according to her current state. On the one hand, populations are large and a deviation by a single player may have little effect on the aggregate behavior of the whole population at any time. On the other hand, due to the large size of population and the complexity of the dynamic process, it may not be worthwhile for a player to think about the feedback impact of her current strategy to her own future payoff. Therefore, a player may prefer her current optimal strategy to other strategies.

We adopt the definition of a continuous-time best-response dynamic formulated by a constant revision rate and myopic optimization, as in Gilboa and Matsui (1991), Matsui (1989) and Hofbauer (1995). Such dynamic can be viewed as a continuous-time analog to a fictitious play. In the following general result for extensive-form games, we apply the standard generic condition, as described in Section A.2.

Main Result 1: A continuous-time best-response dynamic converges to the set of Nash equilibria in all finite generic extensive-form games of perfect information.

Such continuous-time best-response dynamics have been extensively analyzed in various classes of games in strategic (or normal) form; see, e.g., the books of Cressman (2003) and Sandholm (2010). For extensive-form games of perfect information, Cressman (2003, p. 259) gives an interesting example of a centipede game of length 4 and shows that the subgame perfect 
Nash equilibrium component is not asymptotically stable for a best-response dynamic. Therefore, the set of Nash equilibria in this game is not asymptotically stable. Cressman shows that a trajectory initially in the backwardinduction equilibrium component deviates from the equilibrium component for a period of time. However, at the initial state given by Cressman, the distribution of the local behavior strategy at the final decision node in the example game is entirely on a dominated strategy, which will be corrected in the long run if the final node is reached. Therefore, regardless of whether the final node is reached or not, the dynamic should behave in the long run as in a centipede game of length 3 , and the trajectory should converge to the backward-induction equilibrium component.

"Convergence of best-response dynamics in extensive-form games of perfect information is an open problem," Cressman (2003) writes, "even in the case where there is a unique Nash equilibrium component." We dispense with this condition, and prove that a best-response dynamic converges to a Nash equilibrium component at the exponential decay rate, in any finite generic extensive-form game of perfect information.

Cressman and Schlag (1998) show in a finite generic extensive-form game of perfect information that a replicator dynamic converges to a Nash equilibrium. However, this result can only be applied to an interior dynamic. For any replicator dynamic with an initial state in a pure strategy profile, the trajectory will stay at the initial state forever.

Unlike a replicator dynamic, a best-response dynamic is not a regular selection dynamic, and the solution trajectory may not be unique. To be more specific, the strategy adjustment rate may not be continuous, and there may be multiple best-reply strategies for a player at some time. When the state is in the basin of attraction to a Nash equilibrium component, the projection distribution of some player's strategies may remain unchanged in a subgame off the equilibrium path. If that distribution generates multiple best replies from other players, the strategy distribution of those players may "drift" freely. (See the game in Figure 2 for an example.) Hence, we cannot guarantee the convergence of an (interior) dynamic to any particular Nash equilibrium in this case.

In our setup, we can only guarantee the convergence of a best response dynamic to a Nash equilibrium component, which is a set of Nash equilibria with the same outcome. In Section 2, we define a quasi-strategy to be a class of pure strategies such that the outcome in the game is the same regardless of the strategies used by the other players. A quasi-strategy only consists of the sequence of moves that the player may be called upon to play. If a player has already deviated from the play through a node $n$ at one of the predecessors of node $n$, then the player knows that that node will not be reached by any 
means. Therefore, she does not need to consider her moves after $n$. From a dynamic point of view, only the moves included in a quasi-strategy matter for the movement of the trajectory. For perfect-information games without chance nodes, a quasi-strategy is also a strategy in the semi-reduced normalform game; see Lemma A.1.

To show that the convergence of the best-response dynamic in Main Result 1 is robust, we consider an approximate best-response dynamic adapted from the $\epsilon$-accessible path defined in Gilboa and Matsui (1991). This is a weaker version of a best-response dynamic, in the sense that a limitation is imposed on the players' ability to recognize the current state. Therefore, the direction at each point of the trajectory may not be a best response to the current state, but rather a best response to a different strategy profile very close to the current state. For this more general dynamic, it is not true that an approximate best-response dynamic always converges to the set of Nash equilibria; see the game in Figure 3 for an example. However, we can show that the trajectory comes close to the set of Nash equilibria most of the time.

Main Result 2: An approximate best-response dynamic converges in a weaker sense to the set of Nash equilibria in all finite generic extensive-form games of perfect information: the state is close to the set of Nash equilibria most of the time.

In Section 4 we will formalize the definition of the approximate bestresponse dynamic. Benaim and Weibull (2003) applied this notion of weak convergence to a stochastic model, and called the time fractions of convergence there the empirical visitation rate. Young (2009) also applied this notion of weak convergence to show that behavior in so-called interactive trial and error learning comes close to a Nash equilibrium most of the time. We prove in our setup that an approximate best-response dynamic converges to the set of Nash equilibria in this weak sense whatever the initial state and whatever the exact change of direction in the specified neighborhood of the current state at any time.

For the technical part, we show that the distribution of plays converges to a single play in a best-response dynamic, and, moreover, that play is an equilibrium path. For an approximate best-response dynamic, we apply induction to the number of decision nodes in our proof. Note that the set of Nash equilibria may change in the induction step. To avoid the discussion on such possible change of the set of Nash equilibria, we show by induction that most of the time the distribution of plays concentrates on a single play, which is an equilibrium path in the game we are studying.

Since the backward-induction equilibrium is traditionally regarded as the 
rational solution in an extensive-form game of perfect information, it is natural to ask whether the (approximate) best-response dynamic converges to the backward-induction equilibrium. The answer to the general situation is no, as we will see in the game in Figure 3. However, in the case where each player can move at only one node, the convergence of an interior dynamic in Main Result 1 is indeed to the backward induction equilibrium. To see this, note that the mixed strategy of a player is a distribution of all moves directed from the same node, say $n$. If, in all subgames after $n$, the projection distribution in the state is close to the backward induction equilibrium in the subgame, then the dominating move at $n$ in the dynamic is the backwardinduction move in the subgame rooted at $n$. This dominating move at $n$ is the direction of the change for this player from then on.

For any finite generic extensive-form game of perfect information, given any initial state we can find an approximate best-response dynamic such that the trajectory converges to the backward induction equilibrium. We observe that when the probability of any non-backward-induction move at some node is small enough, we can then assume that the player plays the backward-induction move at that node at that time in an approximate bestresponse dynamic. We then apply induction to complete the proof.

Gilboa and Matsui (1991) introduce the definitions of a cyclically stable set and a socially stable strategy. These stability notions incorporate the idea that if the perturbation tendency of state recognition is observed, then people are likely to follow this behavior pattern and the dynamic may turn out to be a positive feedback process. Some Nash equilibria in an extensive-form game may not be stable (cyclically or socially) in an approximate best-response dynamic. We show that in any finite generic extensive-form game of perfect information, the backward-induction equilibrium belongs to the cyclically stable set. If each player can move at only one node, then the backwardinduction equilibrium is the only socially stable strategy in the game.

\section{The Model}

We adopt the standard definition of a finite extensive-form game of perfection information; see, e.g., Kreps (1982), Hart (1992) and Ritzberger (2002). The main contribution here is the introduction of the quasi-strategy, i.e., a class of strategies with an equivalent outcome.

Given a set $N$ of finitely many nodes, we define a partial-order binary relation $\prec$ on $N$ that represents precedence. We further suppose an initial node $n^{0}$ as a predecessor of all other nodes in $N$. Such $(N, \prec)$ defines a tree $T$, and we call $n^{0}$ the root of $T$. We define the immediate-predecessor 
function $\psi: N \rightarrow N$ such that

$$
\psi\left(n^{\prime}\right)=\max \left\{n: n \prec n^{\prime}\right\} \forall n^{\prime} \in N \backslash\left\{n^{0}\right\}
$$

and $\psi\left(n^{0}\right)=\emptyset$. Let $\Psi$ be the predecessor function $\Psi: N \rightarrow 2^{N}$ with

$$
\Psi\left(n^{\prime}\right)=\left\{n \in N: n \prec n^{\prime}\right\} .
$$

We denote $\psi^{-1}$ to be the immediate-successor function. Thus, $\psi^{-1}(n)=$ $\left\{n^{\prime} \in N: n=\psi\left(n^{\prime}\right)\right\}$ for all $n$ in $N$. The successor function $\Psi^{-1}$ can be similarly deduced. We call a node $n$ a terminal node if $\psi^{-1}(n)=\emptyset$, and write $N_{t}:=\left\{n \in N, \psi^{-1}(n)=\emptyset\right\}$.

We say that a sequence $\left\{n_{1}, \ldots, n_{i}\right\}$ of nodes is a subplay in the tree $T$ if $n_{j-1}=\psi\left(n_{j}\right)$ for all $1<j \leq i$. If $n_{1}=n^{0}$ and $n_{i} \in N_{t}$, then it is called a play. Denote the set of all plays by $H$.

We define a $k$-player extensive-form game of perfect information on the finite tree $(N, \prec)$. Denote by $\mathcal{N}=\left\{\Lambda^{0}, \Lambda^{1}, \ldots, \Lambda^{k}\right\}$ a partition of $N \backslash N_{t}$, and call it the assignment of decision nodes. The members of $\Lambda^{0}$ are called chance nodes; for each $i \leq k$, the members of $\Lambda^{i}$ are called the nodes of player $i$. Given a node $n \in N$, we put $\lambda(n)$ as the indicator of which player moves on this node. Hence $\lambda(n)=i$, if $n \in \Lambda^{i}$. For chance nodes, define $\tau: \psi^{-1}\left(\Lambda^{0}\right) \rightarrow[0,1]$ to be a probability distribution function such that

$$
\sum_{n^{\prime} \in \psi^{-1}(n)} \tau\left(n^{\prime}\right)=1 \forall n \in \Lambda^{0} .
$$

We define a vector $\mathbf{v}=\left(v^{1}, \ldots, v^{k}\right)$ such that each $v^{i}: H \rightarrow \mathbb{R}$ is a Bernoulli function of player $i$ for all $1 \leq i \leq k$. Since there is an one-to-one correspondence between $N_{t}$ and $H$, we may abuse the notation and write $v^{i}(n)=v^{i}(h)$ when $n \in h \cap N_{t}$. We call the quadruple $(T, \mathcal{N}, \tau, \mathbf{v})$ an extensive-form game $\Gamma$ of perfect information.

For each player $i$, a (pure) strategy $a^{i}$ assigns a successor to each node in $\Lambda^{i}$. Hence $\psi\left(a^{i}(n)\right)=n$ for all $n$ in $\Lambda^{i}$. Denote the set of pure strategies of player $i$ by $A^{i}$, and the set of pure-strategy profiles by $A=\prod_{i=1}^{k} A^{i}$. We denote the probability distribution of play in game $\Gamma$ for a pure strategy profile $a$ to be a function $\rho_{a}: H \rightarrow[0,1]$ with $\sum_{h \in H} \rho_{a}(h)=1$. (Note that $H$ is finite.) Given a node $\bar{n}$, we denote $H_{\bar{n}}:=\{h \in H: \bar{n} \in h\}$ and say that the node $\bar{n}$ is connected (or reached) under a pure strategy profile $a$ if

$$
\sum_{h \in H_{\bar{n}}} \rho_{a}(h)>0 .
$$


When $\Lambda^{0}=\emptyset$, given such a pure strategy profile $a$ in $A$, we can find a play $h=\left\{n_{0}, n_{1}, \ldots, n_{m}\right\}$ such that $\rho_{a}(h)=1, n_{0}=n^{0}$, and

$$
n_{i+1}=a^{\lambda\left(n_{i}\right)}\left(n_{i}\right) \forall 0 \leq i<m
$$

(Hence the last node $n_{m} \in N_{t}$.)

The set of mixed strategies for player $i$ is defined as

$$
X^{i}:=\Delta\left(A^{i}\right)=\left\{\sigma=\left(\sigma^{i}(a)\right)_{a \in A^{i}}: \sigma^{i}(a) \geq 0 \forall a \in A^{i} \text { and } \sum_{a \in A^{i}} \sigma^{i}(a)=1\right\} \text {. }
$$

Hence a mixed strategy $x^{i}$ is a vector of probabilities assigned to each pure strategy in $A^{i}$. The set of mixed-strategy profiles is denoted by $X=\prod_{i=1}^{k} X^{i}$. We call the induced probability distribution of a mixed-strategy profile $x$ over plays in $T$ the outcome of $x$. Note that a pure-strategy profile $a$ generates a payoff vector $\mathbf{u}(a)=\sum_{h \in H} \rho_{a}(h) \mathbf{v}(h)$. We can linearly extend it to a mixed-strategy profile $x$ :

$$
\mathbf{u}(x)=\sum_{a \in \operatorname{supp}(x)}\left(\prod_{a^{i} \in a} x^{i}\left(a^{i}\right)\right) \mathbf{u}(a) .
$$

A mixed-strategy profile $x$ is a Nash equilibrium of the game $\Gamma$ if

$$
u^{i}(x) \geq u^{i}\left(y^{i}, x^{-i}\right)
$$

for every $i \leq k$ and every $y^{i} \in X^{i}$, where $x^{-i}:=\left(x^{j} \mid 1 \leq j \leq k, j \neq i\right)$. We denote by $N E$ the set of all Nash equilibria.

A subtree rooted at a node $n$ is the truncated tree $\left(\Psi^{-1}(n) \cup\{n\}, \prec\right)$. A subgame rooted at node $n$ is the corresponding subtree with the projection assignment of decision nodes and the payoff function. We denote this subgame by $\Gamma_{n}$, and denote the set of all nodes in $\Gamma_{n}$ by $N\left(\Gamma_{n}\right)$. A Nash equilibrium is a backward-induction equilibrium (also called subgame-perfect equilibrium) if it induces a Nash equilibrium in all subgames. Kuhn proved in Kuhn (1953) that there always exists a pure backward-induction equilibrium, constructed from the terminal nodes and going towards the root. In this paper, we consider a generic finite $k$-player game $\Gamma$ in extensive form with perfect information. Under the generic assumption, the backward-induction equilibrium in $\Gamma$ is unique.

For a mixed strategy profile $x$, we say that a node $\bar{n}$ is connected under $x$ if there exists a pure strategy profile $a$ with non-zero probability in $x$ such that node $\bar{n}$ is connected under $a$. A realized play of a Nash equilibrium is also called an equilibrium path. 
In an extensive-form game, two different pure strategies for the same player always induce the same probability distributions over plays, if they differ only at disconnected nodes (cf. Proposition 4.1 in Ritzberger (2002)). This observation suggests a lower-dimensional representation of an extensiveform game. We call two pure strategies $a_{1}^{i}$ and $a_{2}^{i}$ for player $i$ outcome equivalent and write $a_{1}^{i} \sim a_{2}^{i}$ if, with every combination $a^{-i}$ of strategies for the other players, the outcomes generated by these two strategies are always the same, i.e.,

$$
\rho_{\left(a_{1}^{i}, a^{-i}\right)}(h)=\rho_{\left(a_{2}^{i}, a^{-i}\right)}(h) \forall h \in H \forall a^{-i} \in A^{-i} .
$$

Such a relationship of outcome equivalence generates for each player $i$ a partition $B^{i}$ of the set $A^{i}$. That means:

1. The union of all sets in $B^{i}$ equals $A^{i}$.

2. Given any $b^{i}$ in $B^{i}$, for any two strategies $a_{1}^{i}, a_{2}^{i} \in b^{i}$, (2.4) holds.

Thus, each $b^{i}$ is an equivalence class, and we call $B^{i}$ the set of pure quasistrategies of player $i$, and the set of pure quasi-strategy profiles is defined as $B:=\prod_{i=1}^{k} B^{i}$. Given a pure quasi-strategy profile $b=\left(b^{1}, \ldots, b^{k}\right)$, we can find a pure strategy profile $a=\left(a^{1}, a^{2}, \ldots, a^{k}\right)$ with $a^{i} \in b^{i}$ for all $1 \leq i \leq k$, and we define the payoff vector of profile $b$ as $\left.\mathbf{u}(b):=\sum_{h \in H}\left(\rho_{a}(h)\right) v(h)\right)$. The set of mixed quasi-strategies and the payoff vector of a mixed quasistrategy profile can be defined analogously to (2.2) and (2.3), respectively. Nash equilibria can also be defined using quasi-strategies. In this paper we use quasi-strategies to define the state space. When there is no ambiguity, we may simply refer to a quasi-strategy as a strategy, and denote the set of mixed quasi-strategies of player $i$ by $X^{i}$.

For instance, given the game $\bar{\Gamma}$ in Figure 1, a pure strategy of player I that includes the move $\alpha_{1}$ at the root must also specify the move she would play at the bottom node. We do not, however, specify the move at the bottom node for a quasi-strategy, as it is impossible to reach the bottom node in that case. Hence, in this one-player game, there are only three quasi-strategies corresponding to $\alpha_{1}, \alpha_{2}$, and $\alpha_{3}$, respectively, in our framework. See Appendix A.1 for a comparison between the partition generated from outcome equivalence and the standard representation of reduced normal form for an extensive-form game: outcome equivalence is defined on the realization of outcome, while a reduced normal-form strategy concerns the payoff equivalence.

If we would like to emphasize that a notation is with respect to a game $G$, then we add $(G)$ after the notation, e.g., $N(G), N_{t}(G)$, etc. 


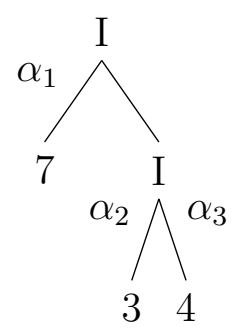

Figure 1: Game $\bar{\Gamma}$

\section{Continuous-Time Best Response Dynamics}

Given a player $i$ in a $k$-player extensive-form game $\Gamma$ of perfect information, for any mixed quasi-strategy profile

$$
x=\left(x^{i}\right)_{1 \leq i \leq k} \in \times_{1 \leq i \leq k} \Delta\left(B^{i}\right),
$$

let $B R^{i}(x)$ be the set of best responses in a pure quasi-strategy for player $i$, i.e.,

$$
B R^{i}(x)=\arg \max _{b^{i} \in B^{i}} u^{i}\left(b^{i}, x^{-i}\right) .
$$

The sequence $\left(\left(x_{t}^{i}\right)_{1 \leq i \leq k}\right)_{t \in \mathbb{N}}$ is a discrete-time fictitious play if

$$
x_{1}^{i} \in \Delta\left(B^{i}\right) \quad \text { and } \quad x_{t+1}^{i}=\frac{t x_{t}^{i}+g_{t}^{i}}{t+1}
$$

for all $1 \leq i \leq k$ and all $t \geq 1$ where $g_{t}^{i} \in B R^{i}\left(x_{t}\right)$. Cressman proves that a discrete-time fictitious play converges to $N E$ in a finite generic extensiveform game of perfect information; see Theorem 8.2.9 in Cressman (2003).

From (3.1), it follows that $x_{t+1}-x_{t}=\left(g_{t}-x_{t}\right) /(t+1)$. If we equate this difference to $\left.\dot{x}\right|_{t}$, we obtain a nonautonomous system due to the factor $1 /(t+1)$. We can ignore this factor and define the (continuous-time) bestresponse dynamic as a process $\left(x_{t}\right)_{t \geq 0}$ with

$$
\dot{x}^{i}=g^{i}-x^{i}
$$

for some $g^{i} \in B R^{i}(x)$. So $\dot{x}^{i} \in B R^{i}(x)-x^{i}$. Up to a rescaling of time, which does not affect the shape of the trajectory, we reach the so-called continuous fictitious play:

$$
\dot{x}^{i} \in \frac{B R^{i}(x)-x^{i}}{t} .
$$

Note that the two continuous-time dynamic processes above are differential inclusions. Since the best-response correspondence is upper-semi-continuous 
with closed and convex values, a solution through a given initial state exists, though it is not necessarily unique; see, e.g., Aubin and Cellina (1984).

Remark: In the literature, it is also common that $B R^{i}(x)$ in (3.1) is defined to be a subset of $X^{i}$. It can be shown that all conclusions in this paper still hold for the more general definition.

We prove the following general convergence result. For a subset $S \subseteq X$ of mixed quasi-strategy profiles, denote the $\epsilon$-neighbourhood of $S$ by $S[\epsilon]$. We apply the supremum norm here. That is, $x \in S[\epsilon]$ if and only if there exists a $y \in S$ such that, for all $i$ with $1 \leq i \leq k$ and all $b^{i} \in B^{i}$,

$$
\left|x^{i}\left(b^{i}\right)-y^{i}\left(b^{i}\right)\right| \leq \epsilon .
$$

Theorem 3.1. Given a finite generic extensive-form game of perfect information without chance nodes, any best-response dynamic $\left(x_{t}\right)_{t}$ or continuoustime fictitious play converges to a Nash equilibrium component. That is, for any $\epsilon>0$, any initial state $x_{0}$, and a best-response dynamic $\left(x_{t}\right)_{t \geq 0}$, there exists a Nash equilibrium component EC and a time $\tau>0$ such that for all $t>\tau$

$$
x_{t} \in E C[\epsilon] .
$$

Remark 1: A best-response dynamic converges to only one Nash equilibrium component. To see this, take a sufficiently small $\epsilon$ in the above theorem.

Remark 2: For simplicity, we consider only extensive-form games without chance nodes here. If we drop this condition, the result still holds under the generic assumption.

In the extensive-form game $\Gamma$, if a move is included in any best-reply quasi-strategy for only a finite period of time, then in the solution trajectory, $x_{t}(b)$ is decreasing to zero for any quasi-strategy $b$ including that move. Therefore, we only need to focus on a play $\left(n_{1}, n_{2}, \ldots, n_{i}\right)$ such that each move $\left(n_{j}, n_{j+1}\right)$ along the play is part of a best-reply quasi strategy for an infinitely long time, and we call such a play a perpetually realized play. We can show that a perpetually realized play exists and is unique. Therefore, the trajectory of the best-response dynamic converges to the set of strategy profiles that generate that special play. We then prove by contradiction that the perpetually realized play is a path of a Nash equilibrium component.

\subsection{Notations and preliminary results}

Suppose that there are $\kappa:=\left|N_{t}\right|$ terminal nodes in the generic $k$-player extensive-form game $\Gamma$. We enumerate the attached payoffs to each player 
in order, respectively, as $\left(v_{(1)}^{i}, \ldots, v_{(\kappa)}^{i}\right)$ for all $i$ with $1 \leq i \leq k$ such that the superscripts specify the players and $v_{(m)}^{i}>v_{(n)}^{i}$ for all $m<n, 1 \leq i \leq k$. We can find a positive number $\delta$ such that for all $i$ with $1 \leq i \leq k$ for all $j \leq \kappa$

$$
(1-\delta) v_{(j)}^{i}+\delta v_{(1)}^{i}<(1-\delta) v_{(j-1)}^{i}+\delta v_{(\kappa)}^{i}
$$

That is, player $i$ 's preference over terminal nodes remains unchanged, even if there is uncertainty of probability $\delta$ over which terminal node is finally reached. We fix such a $\delta$ for the proof later.

For a pure quasi-strategy $b^{i}$ of player $i$, we can denote its domain as $\bar{N}_{b^{i}}$. Hence $n \in \bar{N}_{b^{i}}$, if, for all nodes $n^{\prime} \in \Psi(n) \cap \Lambda^{i}, b^{i}\left(n^{\prime}\right) \in \Psi(n) \cup\{n\}$. That is, if a player $i$ moves towards a node $n$ from every predecessor node where she could play, then player $i$ should also specify her move at node $n$. Therefore a pure quasi-strategy $b^{i}$ of player $i$ in $\Gamma$ can be represented by a sequence of moves as

$$
S\left(b^{i}\right):=\left(\left(n, b^{i}(n)\right): n \in \bar{N}_{b^{i}}\right) .
$$

Given a player $i$ and a pair of nodes $\left(n_{1}, n_{2}\right)$ with $n_{1} \in \Lambda^{i}$ and $n_{2} \in$ $\psi^{-1}\left(n_{1}\right)$, we denote by $B^{i}\left(n_{1}, n_{2}\right)$ the set including all pure quasi-strategies $b^{i}$ with the property $b^{i}\left(n_{1}\right)=n_{2}$. (Note that some strategy of player $i$ may require $n_{1} \notin \bar{N}_{b^{i}}$.) Given any such pair of nodes above, for a mixed quasistrategy $x^{i}=\left(x^{i}\left(b^{i}\right)\right)_{b^{i} \in B^{i}}\left(\right.$ recall that $x^{i}\left(b^{i}\right) \geq 0$ and $\left.\sum_{b^{i} \in B^{i}} x^{i}\left(b^{i}\right)=1\right)$, define

$$
y\left(n_{1}, n_{2}\right):=\sum_{b^{i} \in B^{i}\left(n_{1}, n_{2}\right)} x^{i}\left(b^{i}\right)
$$

and $y\left(n_{1}\right):=\sum_{n_{2} \in \psi^{-1}\left(n_{1}\right)} y\left(n_{1}, n_{2}\right)$. Thus $y\left(n_{1}\right)$ is the probability that player $i$ plays at node $n_{1}$, and $y\left(n_{1}, n_{2}\right)$ is the probability that player $i$ moves from node $n_{1}$ to node $n_{2}$. Let $x\left(n_{1}, n_{2}\right):=y\left(n_{1}, n_{2}\right) / y\left(n_{1}\right)$, when $y\left(n_{1}\right)>0$. For a node $n_{1}$ with $y\left(n_{1}\right)>0$, we call the distribution $\left(x\left(n_{1}, n_{2}\right)\right)_{n_{2} \in \psi^{-1}\left(n_{1}\right)}$ the local behaviour strategy of player $i$ at node $n_{1}$. For a play $h=\left(n_{0}, n_{1}, \ldots, n_{|h|}\right)$ with $n_{j-1}=\psi\left(n_{j}\right)$ for all $0<j \leq|h|$, we can denote the probability of $h$ followed in game $\Gamma$ for a quasi-strategy profile $x$ by

$$
\rho_{x}(h):=\left\{\begin{array}{cl}
\prod_{0<j \leq|h|} x\left(n_{j-1}, n_{j}\right) & \text { if } x\left(n_{j-1}, n_{j}\right) \text { defined } \forall j \leq|h| \\
0 & \text { otherwise. }
\end{array}\right.
$$

In a dynamic process, we sometimes write $\rho_{t}(h)$ as a shorthand for $\rho_{x_{t}}(h)$.

Given a subtree $\Gamma_{n}$ with root $n$, for a pure quasi-strategy $b^{i}$ of player $i$, the projection quasi-strategy $b^{i}\left(\Gamma_{n}\right)$ in $\Gamma_{n}$ is the subsequence

$$
\left(\left(n^{\prime}, b^{i}\left(n^{\prime}\right)\right): n^{\prime} \in \bar{N}_{b^{i}} \cap N\left(\Gamma_{n}\right)\right)
$$


in $S\left(b^{i}\right)$. (Recall that $N\left(\Gamma_{n}\right)$ contains all nodes in the subtree $\Gamma_{n}$.) We can then define a projection mixed quasi-strategy as a distribution in $\left\{b^{i}\left(\Gamma_{n}\right)\right\}_{b^{i} \in B^{i}}$.

Given a pair of nodes $\left(n, n^{\prime}\right)$ with $n=\psi\left(n^{\prime}\right)$, we define the Lebesgue integral

$$
f\left(n, n^{\prime}\right):=\int_{0}^{\infty} \mathbb{1}_{\left(n, n^{\prime}\right) \in S\left(g_{t}^{i}\right)} \mathrm{d} t
$$

where player $i=\lambda(n)$ and $g_{t}$ is defined in the best-response dynamic (3.2). (Recall that $\mathbb{1}$ is the indicator function.)

We give a straightforward lemma below without proof.

Lemma 3.2. Given a finite generic extensive-form game $\Gamma$ of perfect information, we suppose that $\left(x_{t}\right)_{t>0}$ is a best-response dynamic. We further assume that for a pair of nodes $\left(n, n^{\prime}\right)$ with $\psi\left(n^{\prime}\right)=n$ where player $i$ plays at node $n, n \notin \bar{N}_{g_{t}^{i}}$ or $g_{t}^{i}(n) \neq n^{\prime}$ for all best-response strategies $g_{t}^{i} \in B R^{i}\left(x_{t}\right)$ at time $t$. Then

$$
\left.\dot{x}^{i}\left(\Gamma_{n^{\prime}}\right)\right|_{t}=0, \forall t \geq 0,
$$

where $x^{i}\left(\Gamma_{n^{\prime}}\right)$ is the projection distribution of the quasi-strategy of player $i$ in the subgame $\Gamma_{n^{\prime}}$. If the assumption condition on $\left(n, n^{\prime}\right)$ is valid for all $t \geq 0$, then

$$
x_{t}\left(n_{1}, n_{2}\right)=x_{0}\left(n_{1}, n_{2}\right) e^{-t}
$$

for any pair of nodes $\left(n_{1}, n_{2}\right)$ with $n_{1} \in\left(\Psi^{-1}\left(n^{\prime}\right) \cup\left\{n^{\prime}\right\}\right) \cap \Lambda^{i}$ and $n_{1}=\psi\left(n_{2}\right)$ and for all $t>0$.

If $n \in \bar{N}_{g_{\hat{t}}^{i}}$ and $g_{\tilde{t}}^{i}(n)=n^{\prime}$ at any $\tilde{t} \geq 0$, then for all nodes $\bar{n}$ in $\psi^{-1}(n) \backslash\left\{n^{\prime}\right\}$

$$
\left(\frac{x_{t}\left(n, n^{\prime}\right)}{x_{t}(n, \bar{n})}\right)_{\tilde{t}}^{\prime}=\frac{1}{x_{\tilde{t}}(n, \bar{n})} .
$$

\subsection{Proof of Theorem 3.1}

Under a best-response dynamic, we would like to find and focus on a play that is followed with probability close to 1 in the long run.

Lemma 3.3. For a pair of nodes $\left(\bar{n}, n^{\prime}\right)$ with $\bar{n}=\psi\left(n^{\prime}\right)$ and $f\left(\bar{n}, n^{\prime}\right)=+\infty$, there is a set $N^{\bar{n}} \subseteq \psi^{-1}(\bar{n}) \backslash\left\{n^{\prime}\right\}$ with $f(\bar{n}, n)<+\infty$ for all $n \in N^{\bar{n}}$. Then, for every $\epsilon>0$, there exists a time $t_{\bar{n}}$ such that

$$
\forall t>t_{\bar{n}}, \quad \sum_{n \in N^{\bar{n}}} x_{t}(\bar{n}, n)<\epsilon x_{t}\left(\bar{n}, n^{\prime}\right) .
$$


Proof. Denote the player who moves at node $\bar{n}$ by player $i$. From the definition of $f$ in (3.6), it follows that for every $\mu>0$ there exists a time $t(\mu)$ such that

$$
\sum_{n \in N^{\bar{n}}} \int_{t(\mu)}^{\infty} \mathbb{1}_{\left\{t:(\bar{n}, n) \in S\left(g_{t}^{i}\right)\right\}} \mathrm{d} t<\mu .
$$

The desired result follows from (3.7) and (3.9).

Lemma 3.4. There exists a play $h=\left(n_{1}, \ldots, n_{|h|}\right) \in H$ (with $\left.n_{j-1}=\psi\left(n_{j}\right)\right)$ such that $f\left(n_{i}, n_{i+1}\right)=+\infty$ for all $i$ with $1 \leq i<|h|$. We call such a play a perpetually realized play.

Proof. This follows from the definition of a quasi-strategy (see the definition of $S\left(b^{i}\right)$ in (3.4)) and the finiteness of the game tree.

Lemma 3.5. There is only one perpetually realized play $h_{r}=\left(n_{1}, \ldots, n_{\left|h_{r}\right|}\right)$ in $\Gamma$. Given an $\epsilon>0$, we denote $\hat{\epsilon}=(\delta \epsilon) /(8|N|)$. For every $\epsilon>0$ there exists a time $t(\epsilon)$ such that

$$
\rho_{t}\left(h_{r}\right)>1-\hat{\epsilon}
$$

for all $t>t(\epsilon)$.

Proof. We first prove by contradiction that there is only one perpetually realized play. Assume that there are $j>1$ perpetually realized plays. Then we can find two perpetually realized plays $h^{1}$ and $h^{2}$ such that for some integer $c$, the following properties hold.

1. $n_{i}^{1}=n_{i}^{2}$ for all $1 \leq i \leq c$; we let $n_{c}:=n_{c}^{1}=n_{c}^{2}$.

2. $n_{c+1}^{1} \neq n_{c+1}^{2}$.

3. for any other perpetually realized play $h_{r},\left(h_{r} \backslash\left\{n_{c}\right\}\right) \cap N\left(\Gamma_{n_{c}}\right)=\emptyset$.

(Recall that $N\left(\Gamma_{n_{c}}\right)$ denotes the set of all nodes in the subgame rooted at node $n_{c}$.) From Lemma 3.3, we know that there exists a finite time $\bar{t}$ such that

$$
\forall t>\bar{t}, \forall j=\{1,2\}, \forall i \geq c, x_{t}\left(n_{i}^{j}, n_{i+1}^{j}\right)>1-\hat{\epsilon} .
$$

Denote the player who moves at node $n_{c}$ by player $l$. For any $t>\bar{t}$, for a pure quasi-strategy $b^{l}$ in $B^{l}$ with $b^{l}\left(n_{i}^{1}\right)=n_{i+1}^{1}$ for all $n_{i}^{1} \in \Lambda^{l}$,

$$
N \hat{\epsilon} v_{(\kappa)}^{l}+(1-N \hat{\epsilon}) v^{l}\left(h^{1}\right)<u^{l}\left(\left(b^{l}, x_{t}^{-l}\right)\left(\Gamma_{n_{c}}\right)\right)<N \hat{\epsilon} v_{(1)}^{l}+(1-N \hat{\epsilon}) v^{l}\left(h^{1}\right),
$$

where $u^{1}\left(b^{l}, x_{t}^{-l}\left(\Gamma_{n_{c}}\right)\right)$ is the expected payoff of player $l$ for strategy $b^{l}$ at time $t$ conditional on reaching node $n_{c}$. 
Similarly, for any $t>\bar{t}$, for a pure quasi-strategy $\tilde{b}^{l}$ in $B^{l}$ with $\tilde{b}^{l}\left(n_{i}^{2}\right)=$ $n_{i+1}^{2}$ for all $n_{i}^{2} \in \Lambda^{l}$,

$$
N \hat{\epsilon} v_{(\kappa)}^{l}+(1-N \hat{\epsilon}) v^{l}\left(h^{2}\right)<u^{l}\left(\left(\tilde{b}^{l}, x_{t}^{-l}\right)\left(\Gamma_{n_{c}}\right)\right)<N \hat{\epsilon} v_{(1)}^{l}+(1-N \hat{\epsilon}) v^{l}\left(h^{2}\right) .
$$

Since $N \hat{\epsilon}<\delta$, we infer from (3.12), (3.13), and (3.3) that

$$
u^{l}\left(b^{l}, x_{t}^{-l}\left(\Gamma_{n_{c}}\right)\right) \neq u^{l}\left(\tilde{b}^{l}, x_{t}^{-l}\left(\Gamma_{n_{c}}\right)\right)
$$

for all $t>\bar{t}$. Hence, the definition of best-reply strategy $g_{t}^{i}$ requires that $f\left(n_{c}, n_{c}^{1}\right)=+\infty$ and $f\left(n_{c}, n_{c}^{2}\right)=+\infty$ cannot both hold. Therefore there are at most $j-1$ perpetually realized plays, a contradiction.

If there is only one perpetually realized play, then (3.11) follows straightforwardly.

Lemma 3.6. Given any $\epsilon<1 / 2^{|N|}$, for the perpetually realised play $h_{r}=$ $\left(n_{1}, \ldots, n_{\left|h_{r}\right|}\right)$, there are infinitely many times $t>t(\epsilon)$, where $t(\epsilon)$ is defined in Lemma 3.5, such that

$$
\left.\dot{x}\left(n_{j}, n_{j+1}\right)\right|_{t} \geq 0 \forall j \text { with } 1 \leq j<\left|h_{r}\right| .
$$

Proof. We apply Lemma A.4, which considers a more general definition of a $\mu$-dynamic. A best-response dynamic is a 0 -dynamic. For the formal definition of a $\mu$-dynamic, see to Definition 4.1.

Lemmata A.2 and A.3 in the Appendix show that if a play $h$ is not an equilibrium path, then there exists at least one node $n$ in $h$ such that, in any state where all players follow the play $h$, a deviation from the node $n$ can give the player who plays at $n$ an extra payoff bounded away from zero.

We show below that if the distribution of the outcome in any state is close enough to and moving towards a single play, then that play is an equilibrium path.

For the $k$-player game $\Gamma$, let

$$
c:=\min _{i=1}^{k}\left\{\min _{l=1}^{\kappa-1}\left\{v_{(l)}^{i}-v_{(l+1)}^{i}\right\}\right\},
$$

and

$$
\bar{\epsilon}:=\min \left\{c, \frac{c}{\max _{i=1}^{k}\left(2 v_{(1)}^{i}-2 v_{(\kappa)}^{i}\right)}\right\} .
$$


Lemma 3.7. For any

$$
\epsilon<\bar{\epsilon}
$$

if both (3.11) and (3.14) hold for the perpetually realized play $h_{r}$ in $\Gamma$ at some time $t$, then $h_{r}$ is an equilibrium path in $\Gamma$.

Proof. Given the play $h_{r}=\left(n_{1}, \ldots, n_{\left|h_{r}\right|}\right)$, we define $X\left(h_{r}\right)$ to be the set of mixed strategy profiles $x$ with $x\left(n_{j}, n_{j+1}\right)=1$ for all $1 \leq j<\left|h_{r}\right|$. (Recall that $x\left(n_{j}, n_{j+1}\right)$ is the probability of moving from $n_{j}$ to $n_{j+1}$ in the local behavior strategy at node $n_{j}$.)

We consider a time $t$ when both (3.11) and (3.14) hold. We modify $x_{t}$ to an $\hat{x} \in X\left(h_{r}\right)$ such that

1. $\hat{x}\left(n_{j}, n_{j+1}\right)=1$ for all $1 \leq j<\left|h_{r}\right|$;

2. $\hat{x}\left(n^{\prime}, n^{\prime \prime}\right)=x_{t}\left(n^{\prime}, n^{\prime \prime}\right)$ for all $\left(n^{\prime}, n^{\prime \prime}\right)$ with $n^{\prime} \notin h_{r}, x_{t}\left(n^{\prime}, n^{\prime \prime}\right)$ defined, and $\lambda\left(n^{\prime}\right) \neq \lambda\left(\psi^{\bar{q}}\left(n^{\prime}\right)\right)$ in which $\bar{q}:=\min \left\{q: \psi^{q}\left(n^{\prime}\right) \in h_{r}\right\}$. (Roughly speaking, the last condition above means that the player who moves at node $n^{\prime}$ is not the player who can deviate from $h_{r}$ towards node $n^{\prime}$.)

Therefore the behavior strategy of each player in $\hat{x}$ is well defined, and $\hat{x}$ is in $X\left(h_{r}\right)$.

Suppose $h_{r}$ is not an equilibrium path; we pick a deviation node $n_{j}$ in $h_{r}$ as defined in Lemma A.2, and denote the player who moves at $n_{j}$ by player $i$. We then take a pure quasi-strategy $g_{\hat{x}^{-i}}$ of player $i$, defined in (A.3), which is a best reply of player $i$ against $\hat{x}^{-i}$ under the constraint of a move at node $n_{j}$ towards a node different from $n_{j+1}$. From (3.11), we may infer

$$
\begin{gathered}
u^{i}\left(x_{t}\right)<\epsilon v_{(1)}^{i}+(1-\epsilon) v^{i}\left(h_{r}\right) ; \\
u^{i}\left(g_{\hat{x}^{-i}}, x_{t}^{-1}\right)>\epsilon v_{(\kappa)}^{i}+(1-\epsilon) \hat{u}\left(\hat{x}^{-i}\right),
\end{gathered}
$$

where $\hat{u}\left(\hat{x}^{-i}\right)$ is the payoff to player $i$ for $\left(g_{\hat{x}^{-i}}, \hat{x}^{-i}\right)$. We also deduce from (A.5) in Lemma A.3 and (3.15) that

$$
\hat{u}\left(\hat{x}^{-i}\right)-v^{i}\left(h_{r}\right) \geq c .
$$

From (3.16), it follows that

$$
\epsilon<\frac{c}{\left(2 v_{(1)}^{i}-v^{i}(h)-v_{(\kappa)}^{i}\right)}
$$

Therefore

$$
\epsilon\left(v_{(1)}^{i}+\hat{u}\left(\hat{x}^{-i}\right)-v^{i}\left(h_{r}\right)-v_{(\kappa)}^{i}\right)<c .
$$


From (3.17), (3.18), (3.19), and (3.20), it follows that

$$
u^{i}\left(x_{t}\right)<u^{i}\left(g_{\hat{x}^{-i}}, x_{t}^{-1}\right) .
$$

Hence (3.14) does not hold at time $t$, a contradiction.

Rigorously speaking, the convergence of the distribution of plays to an equilibrium path is not equal to the convergence of the trajectory to the corresponding equilibrium component. One needs to show that from some time on, the behavior-strategy distribution off the equilibrium path either confines the state in the basin of attraction to that equilibrium component, or not far away from the projection distribution of a strategy profile in the equilibrium component.

If (3.14) always holds from some time $t$, then it follows from (3.7) and (3.8) that both (3.11) and (3.14) hold for $h$ from that $t$ on. Even if (3.14) is sometimes not true, it is true most of the time and the state is always in the $\epsilon$-neighborhood of the equilibrium component whose path is the perpetually realized play.

Proof of Theorem 3.1. Without loss of generality, we take an

$$
\epsilon<\min \left\{1 / 2^{|N|}, \bar{\epsilon}\right\} .
$$

By Lemmata 3.5, 3.6, and 3.7, there exists a time $\bar{t}>t(\epsilon)$ such that both (3.11) and (3.14) hold for an equilibrium path $h$. Denote the equilibrium component with equilibrium path $h$ by $E C$. Therefore $x_{\bar{t}} \in E C[\hat{\epsilon}]$.

If there exists a time $\tilde{t}$ as the infimum of time $t>\bar{t}$ such that (3.14) does not hold at time $t$, then there must exist a time $\hat{t}$ as the infimum of time $t>\tilde{t}$ such that (3.14) holds at time $t$. Recall that for any edge $\left(n_{j}, n_{j+1}\right)$ in $h$, if $\dot{x}\left(n_{j}, n_{j+1}\right)<0$, then $\dot{\rho}(h)<0$; see Lemma A.4. In that case, by (3.8) as well as (A.8) and (A.9) in the proof of Lemma A.4 (for the case of $\mu=0$ ), we find that

$$
\left.\dot{\rho}(h)\right|_{t}<-(1-2|N| \hat{\epsilon})=-(1-\epsilon / 4)
$$

for all $t$ with $\tilde{t}<t<\hat{t}$. (Recall that the number of nodes in $\Gamma$ is $|N|$.) We may also infer from (3.21) and Lemma 3.5 that

$$
\left(1-\frac{\epsilon}{4}\right) e^{-(\hat{t}-\tilde{t})}>1-\frac{\epsilon}{4}-\hat{\epsilon} .
$$

To see this, note that $\rho_{t}(h)>1-\hat{\epsilon}$ for any $t \in[\tilde{t}, \hat{t}]$, and that for the function $y=(1-\epsilon / 4) e^{-x},\left.\dot{y}\right|_{x}>-(1-\epsilon / 4)$ for all $x>0$. We compare the function $\rho_{t}(h)$ and $y$ and hence reach (3.22). 
We then infer from (3.22) and the proposition of the best-response dynamic that

$$
\left|x_{t_{1}}^{i}\left(b^{i}\right)-x_{t_{2}}^{i}\left(b^{i}\right)\right|<\frac{\epsilon}{4}+\hat{\epsilon}<\frac{\epsilon}{2} \forall \tilde{t}<t_{1}<t_{2}<\bar{t} \forall b^{i} \in B^{i} \forall 1 \leq i \leq k .
$$

Therefore $x_{t} \in E C[\epsilon]$ for all $t$ with $\tilde{t} \leq t \leq \hat{t}$. We observe that $x_{\hat{t}} \in E C[\hat{\epsilon}]$.

Comment 1: It is not always true that an interior best-response dynamic converges to a single Nash equilibrium. (An interior dynamic requires that $y_{0}(n)>0$ for all non-terminal nodes $n$ in $\Gamma$; see the definition of $y(n)$ just below (3.5).) Consider the game in Figure 2. Suppose that at time $t=0$,

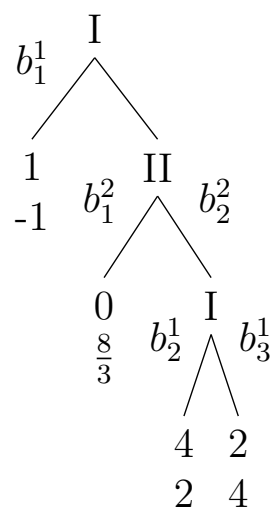

Figure 2: Not converging to one Nash equilibrium

$$
x^{1}\left(b_{1}^{1}\right)<1, \quad \frac{x^{1}\left(b_{2}^{1}\right)}{x^{1}\left(b_{2}^{1}\right)+x^{1}\left(b_{3}^{1}\right)}=\frac{2}{3} \quad 0<x^{2}\left(b_{1}^{2}\right) \leq \frac{1}{8} .
$$

Then $\left\{b_{1}^{1}\right\}=B R^{1}\left(x_{0}\right)$ and $\left\{b_{1}^{2}, b_{2}^{2}\right\}=B R^{2}\left(x_{0}\right)$. We can choose $\left(g_{t}^{2}\right)_{t}$ such that the $\left(x_{t}\right)_{t}$ dynamic has the following properties for all $t \geq 0$.

1. $\left\{b_{1}^{1}\right\}=B R^{1}\left(x_{t}\right)$,

2 .

$$
\frac{x_{t}^{1}\left(b_{2}^{1}\right)}{x_{t}^{1}\left(b_{2}^{1}\right)+x_{t}^{1}\left(b_{3}^{1}\right)}=\frac{2}{3}
$$

(this follows from (3.7)),

3. $\left\{b_{1}^{2}, b_{2}^{2}\right\}=B R^{2}\left(x_{t}\right)$, 
4. $x^{2}\left(b_{2}^{2}\right) \leq 1 / 8$.

In this way, $\left(x_{t}\right)_{t}$ converges to the Nash equilibrium component, but $x_{t}^{2}$ need not be fixed in the dynamic process, and hence $\left(x_{t}\right)_{t}$ need not converge to any particular Nash equilibrium.

Comment 2: The finite time $\tau$ in Theorem 3.1 need not be bounded. See, e.g., the game in Figure 8.3.3 on page 259 in Cressman (2003), which shows that $N E$ is not the maximal attractor for the best-response dynamic. We assume that the initial state of a $\mu$-dynamic is $\left(D,\left(\frac{4}{5}, 0, \frac{1}{5}\right)\right)$. Then, we can let $g_{t}^{1}=D$ and $x_{t}=x_{0}$ until an arbitrary finite time $\bar{t}$. If $g_{\bar{t}}^{1}$ turns to $A A$ and $x_{\bar{t}}^{1}(A A)>0$, then the trajectory enters $N E[\epsilon]$ in bounded time starting from $\bar{t}$.

Comment 3: We use a Lebesgue integral in (3.6) for general dynamic processes. For instance, in the game in Figure 2, we can require that in some period $\left[t_{1}, t_{2}\right], g_{t}^{2}=b_{1}^{2}$ when $t$ is a rational number, and $g_{t}^{2}=b_{2}^{2}$ when $t$ is an irrational number.

Comment 4: Readers may find that the proof above has a similar flavor to the proof in Cressman and Schlag (1998). We show below why we cannot imitate their proof for a best response dynamic process.

1. Their proof is not designed for the convergence to a Nash equilibrium component. For example, in their proof to Lemma 2, Cressman and Schlag (1998) claim that "since $v_{1}$ is a final decision node of $\Gamma^{\prime}$, by the definition of $e_{i}^{1}, u_{1}\left(e_{i}^{1}, f_{i}\right) \geq u_{1}\left(e_{i}, f_{i}\right)$ for any $f_{j} \in F_{2}$." In contrast, in the game in Figure 2 above, we can make $1 / 16 \leq x_{t}^{2}\left(b_{2}^{2}\right) \leq 1 / 8$, and we know that $u^{1}\left(b_{1}^{1}, b_{2}^{2}\right)=1<u^{1}\left(b_{2}^{1}, b_{2}^{2}\right)=4$, and $\lim \sup _{t} x_{t}^{1}\left(b_{2}^{1}\right)=0$. The strategies $b_{1}^{1}, b_{2}^{2}$, and $b_{2}^{1}$ here can be viewed, respectively, as the strategies $e_{i}^{1}, f_{j}$ and $e_{1}$ there. Thus their claim is not always valid for a best-response dynamic.

2. They focus on the integral $\int_{0}^{\infty} x_{t}^{i}\left(b^{i}\right) d t$, which leaves out some basic information about the best-reply propositions in our setup.

Even if $\int_{0}^{\infty} x_{t}^{i}\left(b^{i}\right) d t<\infty$, it is possible that $x^{i}\left(b^{i}\right)>0$ holds less and less frequently, but that $x^{i}\left(b^{i}\right)$ is bounded from below when it does hold. We instead turn to the integral of the period that a strategy is a best response in (3.6).

3. In (3.2), $\dot{x}$ can sometimes be discontinuous, which cannot happen in a replicator dynamic process. 


\section{Approximate Best-Response Dynamics}

Given a mixed strategy profile $x$ and a positive number $\epsilon$, we abuse the notation and write the $\epsilon$-neighbourhood of $x$ as $x[\epsilon]=\{x\}[\epsilon]$.

Definition 4.1. Consider a $k$-player extensive-form game $\Gamma$ of perfect information with the set of pure quasi-strategies $B^{i}$ for each player $i, 1 \leq i \leq k$. We call a continuous-time dynamic process $\left(x_{t}\right)_{t \geq 0}$ a $\mu$-dynamic on $\Gamma$ if every $x_{t}$ is a k-dimensional vector $\left(x_{t}^{i}\right)_{1 \leq i \leq k} \in \times \Delta\left(B^{i}\right)$ and the dynamic satisfies $\dot{x}^{i}=g^{i}-x^{i}$ for some $g^{i} \in B R^{i}(x[\mu])$ for all $i$ with $1 \leq i \leq k$.

In the example below, we show that a $\mu$-dynamic $\left(x_{t}\right)_{t}$ need not converge to the set of Nash equilibria (denoted by $N E$ ), in an extensive-form game of perfect information.

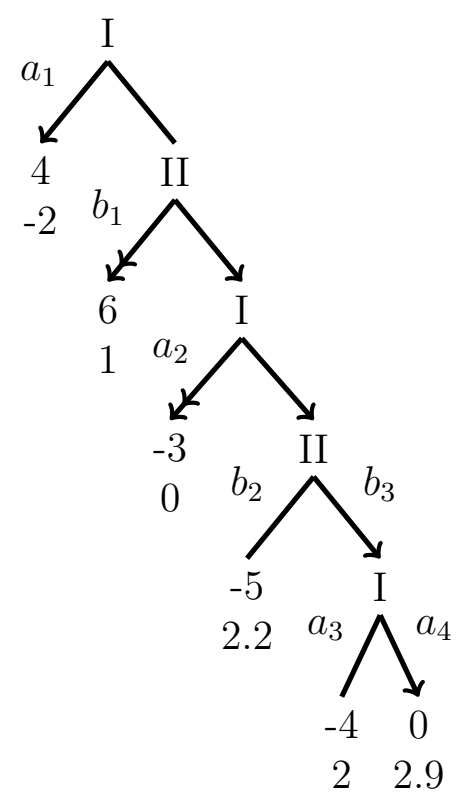

Figure 3: Example in which $\mu$-dynamic need not converge to $N E$

For ease of exposition, the set of best replies for player $i$ at time $t$ is denoted by $B R^{i}\left(x_{t}^{-i}[\mu]\right)$, since it does not depend on the component $x_{t}^{i}$.

In the game in Figure 3, the backward-induction equilibrium is $\left(a_{1}, b_{3}\right)$ and the alternative pure Nash equilibrium is $\left(a_{2}, b_{1}\right)$. (The backward-induction moves are single-arrowed, and the final moves in the alternative equilibrium are double-arrowed.) We denote the corresponding two Nash equilibrium components by $B C$ and $N C$, respectively. If $x_{t}$ ever approaches $N E$, then there exists a time $t_{0}$ such that $x_{t_{0}} \in B C[\mu]$ or $x_{t_{0}} \in N C[\mu]$. Without loss of generality, we let $\mu \leq 1 / 100$. 
1. $x_{t_{0}} \in N C[\mu]$ : we define a mixed quasi-strategy $\tilde{x}^{2}$ of player 2 to be $\tilde{x}^{2}\left(b_{1}\right)=1-\mu$ and $\tilde{x}^{2}\left(b_{3}\right)=\mu$. Then for any $x \in N C, \tilde{x}^{2} \in x^{2}[\mu]$. We assume that

$$
x_{t} \in N C[\mu] \forall t \geq t_{0},
$$

which we show below to be in fact not true for some $\mu$-dynamic. Under the assumption, we can let

$$
g_{t}^{1} \in B R^{1}\left(\tilde{x}^{2}\right) \forall t \geq t_{0}
$$

in a $\mu$-dynamic and hence

$$
g_{t}^{1}=a_{4} \forall t \geq t_{0}
$$

Then there exists a time $t_{1} \geq t_{0}$ such that $x_{t_{1}}^{1}\left(a_{3}\right)+x_{t_{1}}^{1}\left(a_{4}\right)>2 / 3$. Then at some time before $t_{1}, g^{2}$ becomes $b_{3}$, and so $g_{1}$ can still be set as $a_{4}$. Hence, the trajectory is moving away from $N C$ towards $B C$. We then infer that Assumption (4.1) cannot hold for every $\mu$-dynamic.

2. $x_{t_{0}} \in B C[\mu]$ : we define a mixed quasi-strategy $\bar{x}^{1}$ of player 1 to be $\bar{x}^{1}\left(a_{1}\right)=1-\mu$ and $\bar{x}^{1}\left(a_{3}\right)=\mu$. Then for any $x \in B C, \bar{x}^{1} \in x^{1}[\mu]$. We assume that

$$
x_{t} \in B C[\mu] \forall t \geq t_{0},
$$

which we show below to be in fact not true for some $\mu$-dynamic. Under the assumption, we can let

$$
g_{t}^{2} \in B R^{2}\left(\bar{x}^{1}\right)
$$

and hence $g_{t}^{2}=b_{2}$ in a $\mu$-dynamic from $t_{0}$ until the time $t_{1} \geq t_{0}$ when

$$
x_{t_{1}}^{2}\left(b_{2}\right)>3 / 4 \text {. }
$$

We define another mixed quasi-strategy $\hat{x}^{1}$ of player 1 with $\hat{x}^{1}\left(a_{1}\right)=$ $1-\mu$ and $\hat{x}^{1}\left(a_{2}\right)=\mu$. Then for any $x \in B C[\mu], \hat{x}^{1} \in x^{1}[\mu]$. Under Assumption (4.2), we can then let

$$
g_{t}^{2} \in B R^{2}\left(\hat{x}^{1}\right)
$$

and hence $g_{t}^{2}=b^{1}$ from $t_{1}$ until the time $t_{2} \geq t_{1}$ when $x_{t_{1}}^{2}\left(b_{1}\right)>4 / 5$. When $x_{t}^{2}\left(b^{1}\right)>4 / 5, g_{t}^{1} \neq a_{1}$. From (4.3) and (3.7), it follows that $x_{t}^{2}\left(b_{2}\right) / x_{t}^{2}\left(b_{3}\right)>3$ for all $t$ with $t_{1} \leq t \leq t_{2}$. Then at some time before $t_{2}, g^{1}$ becomes $a_{2}$, and so $g_{2}$ can still be set as $b_{1}$. (Note that the best reply of player 2 against $a_{2}$ is $b_{1}$.) Hence, the trajectory is moving away from $B C$ towards $N C$. We then infer that Assumption (4.2) cannot hold for every $\mu$-dynamic. 
From another direction, we can show why the proof of Theorem 3.1 cannot be applied to all approximate best-response dynamic processes. We check Lemma 3.5 for the game in Figure 3. In a best-response dynamic (0-dynamic), if $f\left(a^{4}\right)=+\infty$ ( $f$ is defined in (3.6)), then

$$
\lim _{t \rightarrow \infty} \frac{x_{t}^{1}\left(a_{3}\right)}{x_{t}^{1}\left(a_{4}\right)}=0 .
$$

Therefore the best reply of player 2 cannot be $b_{2}$ after a sufficiently long time, and so the play with the last move $b_{2}$ cannot be a perpetually realized play. Given a $\mu$-dynamic that transits between $N C[\epsilon]$ and $B C[\epsilon]$ infinitely often, we find that both the plays with the last move $b_{2}$ and $a_{4}$, respectively, are perpetually realized plays. This is because when $x^{1}\left(a_{4}\right)<\mu$, a $\mu$-perturbation on $x^{1}\left(a_{3}\right)$ may decide whether $b^{2}$ or $b^{3}$ is the current best reply of player 2 .

In the game in Figure 3, although the $\mu$-dynamic $\left(x_{t}\right)_{t}$ does not converge to $N E$, the relative proportion of the time spent outside $N E$ is small in the long run, if $\mu$ is small. That means that most of the time the state is very close to some Nash equilibrium component, and if it transits to another component, then the transition time is very small compared with the period when it is close to a component.

For simplicity, we only consider extensive-form games without chance nodes here. If we were to drop this condition, the result would still hold under the generic assumption. Recall that an interior dynamic requires $y_{0}(n)>0$ in the initial state for all non-terminal nodes $n$ in $\Gamma$.

Theorem 4.2. Given any finite generic extensive-form game $\Gamma$ of perfect information without chance nodes, for every $\epsilon>0$ there exists a number $\mu(\epsilon)>0$ and $T(\epsilon)>0$ such that for every interior $\mu(\epsilon)$-dynamic $\left(x_{t}\right)_{t}$ on $\Gamma$ with any initial state $x_{0}$

$$
\frac{1}{T} \int_{0}^{T} \mathbb{1}_{\{t: x t \in N E[\epsilon]\}} \mathrm{d} t \geq 1-\epsilon
$$

for all $T \geq T(\epsilon)$.

From this theorem, which will be proved in the Appendix, we derive the following main theorem.

Theorem 4.3. Given any finite generic extensive-form game $\Gamma$ of perfect information without chance nodes, for every $\epsilon>0$ there exists a number $\mu(\epsilon)>0$ such that for every $\mu(\epsilon)$-dynamic $\left(x_{t}\right)_{t}$ on $\Gamma$ with any initial state $x_{0}$

$$
\lim _{T \rightarrow \infty}\left(\frac{1}{T} \int_{0}^{T} \mathbb{1}_{\left\{t: x_{t} \in N E[\epsilon]\right\}} \mathrm{d} t\right) \geq 1-\epsilon
$$


Proof. If the $\mu$-dynamic is an interior dynamic, then the desired result follows from Theorem 4.2.

If the $\mu$-dynamic is not an interior dynamic, we denote the set $N_{y}:=$ $\left\{n \in N_{d}: \exists t: y_{t}(n)>0\right\}$, where $N_{d}$ is the set of all non-terminal nodes in $\Gamma$. So there exists a finite time $\bar{t}$ such that $y_{t}(n)>0$ for all $n \in N_{y}$ and all $t \geq \bar{t}$. We remove from $\Gamma$ all non-terminal nodes (and the subgames rooted there) not in $N_{y}$, and apply Theorem 4.2 in the resulting game from time $\bar{t}$.

\section{Convergence to the Backward-Induction Equilibrium Component}

Given an extensive-form game of perfect information, we denote the backwardinduction equilibrium component in the game by $B C$. Denote the backwardinduction equilibrium by $B I E$. We show below that if each player can move at only one node in the game, then an interior $\mu$-dynamic with sufficiently small $\mu$ always converges to $B C$.

Theorem 5.1. Let us suppose that in a finite generic extensive-form game $\Gamma$ of perfect information, every player can move at only one node. Then, for every $\epsilon>0$ there exists a number $\mu(\epsilon)>0$ and $T(\epsilon)>0$ such that for every interior $\mu(\epsilon)$-dynamic $\left(x_{t}\right)_{t}$ on $\Gamma$ with any initial state $x_{0}$

$$
x_{t} \in B I E[\epsilon]
$$

for all $t \geq T(\epsilon)$.

Remark 1: This theorem can be applied to the case where $\mu=0$, i.e., a best-response dynamic.

Remark 2: Note that the $\mu$-dynamic converges to the (exact) backwardinduction equilibrium, which is stronger than the convergence to the backwardinduction equilibrium component.

Given an extensive-form game of perfect information, the convergence of a $\mu$-dynamic with small $\mu$ to the backward-induction equilibrium is always possible.

Theorem 5.2. Given a finite generic extensive-form game $\Gamma$ of perfect information and $\epsilon>0$, there exists a number $T(\epsilon)>0$ such that for any initial state $x_{0}$ and any $\mu>0$, there exists a $\mu$-dynamic $\left(x_{t}\right)_{t}$ with the property

$$
x_{t} \in B I E[\epsilon]
$$

for all $t>T(\epsilon)$. 
Comment 1: One may be tempted to prove the following claim. Given a finite generic extensive-form game $\Gamma$ of perfect information and $\epsilon>0$, there exists a number $T(\epsilon)>0$ such that for any initial state $x_{0}$, there exists a best-response dynamic $\left(x_{t}\right)_{t}$ with the property $x_{t} \in B C[\epsilon]$ for all $t>T(\epsilon)$. However, one can give a counterexample of this claim in the game $\Gamma$ in Figure 3. Consider an initial state $x_{0}=\left(\hat{x}^{1}, \hat{x}^{2}\right)$ with $\hat{x}^{2}\left(b_{2}\right)>3 / 4, \hat{x}^{1}\left(a_{1}\right)=99 / 100$ and $\hat{x}^{1}\left(a_{2}\right)=99 / 10000$. Following the analysis of $\hat{x}^{1}$ in Step 2 for $\Gamma$ (the game in Figure 3), we find that the trajectory of the best-response dynamic converges to the non-backward-induction equilibrium component.

Comment 2: The convergence property in Theorem 5.2 need not hold for the non-backward-induction equilibrium component. Consider Theorem 5.1 and any extensive-form game of perfect information with more than one pure Nash equilibrium where each player can move at only one node.

\section{Further Comments}

1. Noldeke and Samuelson (1992) study a model of evolutionary processes in extensive-form games, and they characterize each player by her strategy and conjecture. Here a conjecture is essentially the belief of the player on the local behavior strategy at all unreached decision nodes. A best response of a player is against the behavior-strategy distribution at all reached decision nodes and her conjecture at all other decision nodes. At any time, all players can observe the behavior-strategy distribution at all reached decision nodes.

If we apply such a characterization in our model, then we only need to consider non-interior dynamics. This is because a conjecture is irrelevant in the case of interior dynamics, and once a node is reached at any time $t$, it will be reached at all finite times after $t$. For a non-interior dynamic, by the similar argument in the proof of Theorem 4.3, we can focus on the set of nodes that are eventually reached. From Lemmata A.2 and 3.7, we may infer that for this version of best-response dynamics, the distribution of plays in a finite generic extensive-form game of perfect information converges to an equilibrium path, and the trajectory converges to the set of self-confirming equilibria.

2. One cannot be overoptimistic about the convergence of best-response dynamics. As stated in Hart and Mas-Colell (2005) and Hofbauer and Sigmund (1998, Theorem 8.6.1), there is no natural or reasonable dynamic process that leads to an equilibrium in every (normal-form) game. 
3. For a $\mu$-dynamic in this paper, we assume that a player may not fully recognize the current state, and his best response is against a point in a $\mu$-neighborhood of the current state. We may also consider another related weak best-response dynamic: a player can always recognize the current state, but cannot fully control her behavior in the sense that the direction of the trajectory can be guaranteed only in a neighborhood of the currently best-response strategy. Therefore, it is a trembling-hand version of the best-response dynamic, and we may formulate it as

$$
\dot{x}^{i} \in\left(B R^{i}(x)\right)[\mu]-x^{i}
$$

for each player $i$ in the game.

Note that the limit of the differential inclusion in (6.1) and the one in Definition 4.1 are the same when $\mu$ decreases to zero. We conjecture that Theorem 4.3 is still true for the dynamics described in (6.1). Note that, for this dynamic, Lemma 3.7 cannot be applied directly, and (3.7) may not hold.

4. In the present paper, we confine our attention to the processes of continuous-time best-response dynamics. Yet one may also study a discretization of such continuous-time dynamics, which we called (stochastic) best-reply dynamics in $\mathrm{Xu}$ (2013). A mixed strategy of a player can be approximated by a large population of individuals where the proportion of individuals playing a pure strategy represents the probability of the pure strategy being played in the mixed strategy. We construct a best-reply dynamic as in Kandori et al. (1993), Kandori and Rob (1995), and Hart (2002): in each period, the revision opportunity arrives independently across individuals and time, with probability bounded from below. Each active individual then myopically replaces her strategy by a currently best reply, when selection occurs; she randomly chooses a strategy, when mutation happens with small probability.

In such a best-reply dynamic, a state is called evolutionarily stable if its long-term relative frequency of occurrence is bounded away from zero as the mutation rate decreases to zero and populations grow to infinity; see Hart (2002). The combined results in Hart (2002) and Gorodeisky (2006) show that the backward-induction equilibrium is the only evolutionarily stable state in a finite generic extensive-form game of perfect information where each player can move at only one node. (Hart calls such a game a gene-normal form game.) We have proved in Theorem 5.1 that the backward-induction equilibrium is the only socially 
stable strategy in this kind of game. The analogy between these two results arises from the fact that the dynamics in (6.1) is an iterative limit, $\lim _{\mu \rightarrow 0} \lim _{m \rightarrow \infty}$, of a best-reply dynamic, where $\mu$ is the mutation rate and $m$ is the population size. (Recall that the limit of the differential inclusion in (6.1) and the one in Definition 4.1 are the same.) The stability result of the backward-induction equilibrium applies to both deterministic and stochastic dynamics, and both continuous-time and discrete-time models. A general definition of stability and a characterization of stability conditions under best-response dynamics is a direction for further research.

\section{A Appendix}

\section{A.1 Terminology of reduced normal form}

There are two other well-known normal-form representations of an extensiveform game; see Ritzberger (2002). A normal-form game is semi-reduced (or pure-strategy reduced or quasi-reduced) if for all pairs $\left(s_{1}^{i}, s_{2}^{i}\right)$ of strategies of player $i$ and for all players $i=1, \ldots, k$,

$$
\mathbf{u}\left(s_{1}^{i}, s^{-i}\right)=\mathbf{u}\left(s_{2}^{i}, s^{-i}\right), \forall s^{-i} \Rightarrow s_{1}^{i}=s_{2}^{i} .
$$

A normal-form game is mixed-strategy reduced (or simply reduced) if for all strategies $s^{i}$ of player $i$ and for all players $i=1, \ldots, k$,

$$
\mathbf{u}\left(s^{i}, s^{-i}\right)=\sum_{\bar{s}^{i} \in S^{i}} \sigma^{i}\left(\bar{s}^{i}\right) \mathbf{u}\left(\bar{s}^{i}, s^{-i}\right), \forall s^{-i} \Rightarrow \sigma^{i}\left(s^{i}\right)=1 .
$$

That is, no $s^{i}$ is payoff equivalent to any convex combination of the elements in $S^{i} \backslash\left\{s^{i}\right\}$. (Recall $\sigma^{i}$ in (2.2).) Given an extensive-form game $\Gamma$, we denote the set of strategies for player $i$ in semi-reduced normal form and mixedstrategy reduced normal form by $S^{i}$ and $M^{i}$, respectively. Recall that the set of quasi-strategies for player $i$ is $B^{i}$. From their definitions, we could let $B^{i} \supseteq S^{i} \supseteq M^{i}$ for any player $i$ in any extensive-form game $\Gamma$.

Recall that $\Lambda^{0}=\emptyset$ implies no chance node.

Lemma A.1. Given a generic extensive-form game of perfect information with $\Lambda^{0}=\emptyset$, for each player $i, B^{i} \supseteq S^{i}$ implies that $B^{i}=S^{i}$.

Proof. When $\Lambda^{0}=\emptyset$, for every player $i$ and every two different quasistrategies $b_{1}^{i}, b_{2}^{i} \in B^{i}$, there exist two different plays $h_{1}, h_{2}$ and one strategy combination $b^{-i}$ of other players such that

$$
\rho_{\left(b_{1}^{i}, b^{-i}\right)}\left(h_{1}\right)=\rho_{\left(b_{2}^{i}, b^{-i}\right)}\left(h_{2}\right)=1,
$$


i.e., $\left(b_{1}^{i}, b^{-i}\right)$ and $\left(b_{2}^{i}, b^{-i}\right)$ generate different plays $h_{1}$ and $h_{2}$, respectively, in $\Gamma$. (Rigorously, given a quasi-strategy profile $b=\left(b^{1}, \ldots, b^{k}\right)$ and a pure strategy profile $a=\left(a^{1}, \ldots, a^{k}\right)$ such that $a^{i} \in b^{i}$ for all $i$ with $1 \leq i \leq k$, we have $\rho_{b}(h)=\rho_{a}(h)$ for all plays $h$ in $H$.) Since $\Gamma$ is generic, it follows that

$$
\mathbf{u}\left(b_{1}^{i}, b^{-i}\right) \neq \mathbf{u}\left(b_{2}^{i}, b^{-i}\right) .
$$

This is true for every pair of quasi-strategies $b_{1}^{i}, b_{2}^{i} \in B^{i}$. Recall that the set of quasi-strategies is a partition of pure strategies, and we reach the conclusion $B^{i}=S^{i}$.

Even if $\Lambda^{0}=\emptyset$, it is not true that $B^{i}=M^{i}$ for all players $i$ in all generic extensive-form games of perfect information. In the one-player game $\Gamma^{\prime}$ below, we denote $B^{1}=\left\{b_{1}, b_{2}, b_{3}\right\}$ where $u^{1}\left(b_{1}\right)=3, u^{1}\left(b_{2}\right)=0$ and $u^{1}\left(b_{3}\right)=6$. However, strategy $b_{1}$ can be replaced by a mixed strategy $x^{1}=$ $\left\{\sigma^{1}\left(b_{1}\right)=0, \sigma^{1}\left(b_{2}\right)=1 / 2, \sigma^{1}\left(b_{3}\right)=1 / 2\right\}$. Thus, $M^{1} \neq B^{1}$.

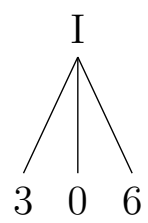

Figure 4: $\Gamma^{\prime}$ with $B^{i} \neq M^{i}$

For the general case without the constraint on $\Lambda$, the conclusion in Lemma A.1 is no longer true. In the one-player game $\Gamma^{\prime \prime}$ below, we denote $B^{1}=$ $\left\{b_{1}, b_{2}\right\}$. The strategies $b_{1}$ and $b_{2}$ lead to the same expected payoff, and hence one can be replaced by the other. Hence $B^{1} \neq S^{1}$.

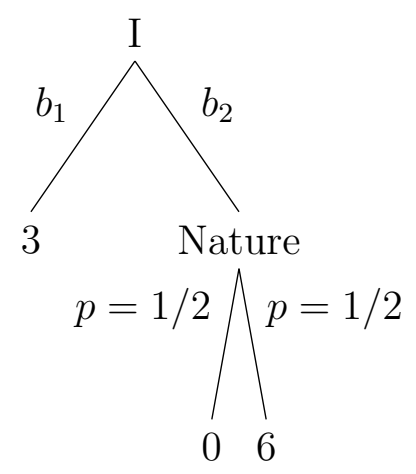

Figure 5: $\Gamma^{\prime \prime}$ with $B^{i} \neq S^{i}$ 


\section{A.2 Generic assumption}

We introduce the generic assumption to rule out any payoff tie that may arise from any backward-induction process in our model. The definition of a generic game discussed here is from Cressman (2003).

An extensive-form game $\Gamma$ is generic if no two pure strategy profiles that yield different outcomes generate the same payoff for any player. For extensive-form games without chance nodes, this is equivalent to the property that no two terminal nodes have the same payoff for any player. To put it formally, given an extensive-form game $\Gamma$, the set of all possible payoffs assigned to terminal nodes for which $\Gamma$ is generic is a set whose complement is a closed set with Lebesgue measure zero.

\section{A.3 Preliminary lemmas for Theorem 3.1}

Given a play $h=\left(n_{1}, \ldots, n_{|h|}\right)$, we define $X(h)$ to be the set of mixed strategy profiles $x$ with $x\left(n_{j}, n_{j+1}\right)=1$ for all $1 \leq j<|h|$. (Recall that $x\left(n_{j}, n_{j+1}\right)$ is the probability of moving from $n_{j}$ to $n_{j+1}$ in the local behavior strategy at node $n_{j}$.)

Lemma A.2. If a play $h=\left(n_{1}, \ldots, n_{|h|}\right)$ is not an equilibrium path in $\Gamma$, then there exists one node $n_{j}$ in $h$ such that for any mixed quasi-strategy profile $x$ in $X(h)$, there exists a pure quasi-strategy $b^{i} \in B^{i}$ such that

$$
b^{i}\left(n_{j}\right) \neq n_{j+1} \text { and } u^{i}\left(b^{i}, x^{-i}\right)>u^{i}(x)=v^{i}(h)
$$

where $i=\lambda\left(n_{j}\right)$. We call such an $n_{j}$ a deviation node.

Proof. Note that each Nash equilibrium component of $\Gamma$ consists of a pure strategy Nash equilibrium together with other Nash equilibria with the same equilibrium path.

We prove the desired conclusion by contradiction. For each node $n_{j}$ in $h$ with $j<|h|-1$, we transform the subgame $\Gamma_{n_{j}}$ to a game $\Gamma^{n_{j}}$ by replacing the node $n_{j+1}$ with a terminal node $n_{h}$ that has the same payoff vector as for the terminal node $n_{|h|}$ in the original play $h$. (Hence the subgame $\Gamma_{n_{j+1}}$ is not included in $\Gamma^{n_{j}}$.) Note that $\Gamma^{n_{|h|-1}}=\Gamma_{n_{|h|-1}}$.

If Assumption (A.1) is not true, then for any $n_{j}$ and the generated game $\Gamma^{n_{j}}$ there exists a distribution $x^{-\lambda\left(n_{j}\right)}\left(\Gamma^{n_{j}}\right)$ such that the best reply of player $\lambda\left(n_{j}\right)$ can be a pure quasi-strategy of the move $\left(n_{j}, n_{h}\right)$. Back to the original game $\Gamma$, for each node $n \notin h$ there exists a unique $j$ such that $n \in \Gamma^{n_{j}}$. We generate a mixed quasi-strategy $\bar{x}$ from $\left(x^{-\lambda\left(n_{j}\right)}\left(\Gamma^{n_{j}}\right)\right)_{j<|h|}$ such that

1. $\bar{x} \in X(h)$; 
2. for every $n \notin h$, the local behavior strategy of $\bar{x}$ at node $n$ agrees with the one in $x^{-\lambda\left(n_{j}\right)}\left(\Gamma^{n_{j}}\right)$ where $n$ is in $\Gamma^{n_{j}}$.

In this way, we can see that play $h$ is the path of a Nash equilibrium $\bar{x}$ in $\Gamma$, a contradiction.

Given a node $n_{j}$ in a play $h=\left(n_{1}, \ldots, n_{\left|h_{r}\right|}\right)$, we denote

$$
B_{n_{j}}:=\left\{b^{\lambda\left(n_{j}\right)} \in B^{\lambda\left(n_{j}\right)}: n_{j} \in \bar{N}_{b^{\lambda\left(n_{j}\right)}} \text { and } b^{\lambda\left(n_{j}\right)}\left(n_{j}\right) \neq n_{j+1}\right\} .
$$

Suppose that player $i$ plays at node $n_{j}$. For every $x^{-i}$ in $X(h)^{-i}$, let $g_{x^{-i}}\left(n_{j}\right)$ be a pure quasi-strategy of player $i$ that is a best response within $B_{n_{j}}$ for the strategy profile $x^{-i}$, i.e.,

$$
g_{x^{-i}}\left(n_{j}\right) \in \underset{b^{i} \in B_{n_{j}}}{\operatorname{argmax}} u^{i}\left(b^{i}, x^{-i}\right) .
$$

We may abuse the notation and drop $\left(n_{j}\right)$ in $g_{x^{-i}}\left(n_{j}\right)$. Let

$$
\hat{u}\left(x^{-i}\right):=\max _{b^{i} \in B_{n_{j}}} u^{i}\left(b^{i}, x^{-i}\right),
$$

and $\hat{u}\left(x^{-i}\right)=u^{i}\left(g_{x^{-i}}, x^{-1}\right)$.

Lemma A.3. We consider a $k$-player generic extensive-form game $\Gamma$ of perfect information with $\kappa$ terminal nodes. If a play $\bar{h}=\left(n_{1}, \ldots, n_{|\bar{h}|}\right)$ is not an equilibrium path in $\Gamma$, then we take a deviation node $n_{j}$ defined in Lemma A.2, and suppose player $i$ moves at node $n_{j}$. Then,

$$
\min _{x \in X(\bar{h})}\left(\hat{u}\left(x^{-i}\right)-v^{i}(\bar{h})\right) \geq \min _{i=1}^{k}\left\{\min _{l=1}^{\kappa-1}\left\{v_{(l)}^{i}-v_{(l+1)}^{i}\right\}\right\}
$$

where $\hat{u}\left(x^{-1}\right)$ is defined in (A.4) with respect to $n_{j}$ in $\bar{h}$, and $v_{(l)}^{i}$ is defined as in Section 3.1.

Note that it is straightforward to see that the left-hand side in (A.5) is bounded away from 0 . We define a function $f^{\prime}: X(\bar{h})^{-i} \rightarrow \mathbb{R}$ such that $f^{\prime}\left(x^{-i}\right)=\hat{u}\left(x^{-i}\right)-v^{i}(\bar{h})$. From Lemma A.2, $f^{\prime}\left(x^{-i}\right)>0$ for all $x^{-i}$ in $X(\bar{h})$. Since $f^{\prime}$ is continuous and $X(\bar{h})^{-i}$ is compact, $f^{\prime}$ is bounded away from 0 .

Proof. For every $x$ in $X(\bar{h})$, we define below a sequence $\left(x_{0}^{-i}, x_{1}^{-i}, \ldots, x_{l}^{-i}\right)$ such that

1. $x_{s}^{-i} \in X(\bar{h})^{-1}$ for all $s$ with $0 \leq s \leq l$;

2. $x_{0}^{-i}=x^{-i}$; 
3. $g_{x_{l}^{-i}}=g_{x_{l-1}^{-i}}$

4. $\hat{u}\left(x_{s}^{-i}\right) \geq \hat{u}\left(x_{s+1}^{-i}\right)$ for all $1 \leq s<l$.

Given $x_{s}^{-i}$ with $s \geq 0$, we take a $g_{x_{s}^{-i}}$. If the set

$$
H_{s}:=\left\{h: \rho_{\left(x_{s}^{-i}, g_{x_{s}^{-i}}\right.}(h)>0\right\}
$$

contains multiple elements, we take an $h$ in $\underset{h \in H_{l}}{\operatorname{argmin}} v^{i}(h)$ and denote it by $h_{s}=\left(n_{1}^{s}, \ldots, n_{\left|h_{s}\right|}^{s}\right)$. We now set $x_{s+1}^{-i}$ as follows. For every non-terminal node $n$ not played by player $i$ in the subgame $\Gamma_{n_{j}}$, i.e., in the set $N_{d}\left(\Gamma_{n_{j}}\right) \cap \Gamma^{-i}$, we check the distance of node $n$ from $h_{s}$, i.e., $\min \left\{q: \psi^{q}(n) \in h_{s}\right\}$.

1. If $q=0$, i.e., node $n$ is in the play $h_{s}$, we let $x\left(n, n^{\prime}\right)=1$ where $n^{\prime} \in$ $h_{s} \cap \psi^{-1}(n)$; i.e., it assigns a move along the play $h_{s}$ with probability 1.

2. If $q>0$, i.e., node $n$ is not in the play $h_{s}$, we assign the same local behavior strategy at node $n$ as in $x_{s}^{-1}$, if $n$ and $\psi^{q}(n)$ are played by different players and $x_{s}^{-1}$ requires a move at node $n$.

Therefore, at any node $n$ in $h_{s} \cap \Lambda^{i} \cap N_{d}\left(\Gamma_{n_{j}}\right)$, if we cut the edge from node $n$ to its successor and predecessor nodes in $h_{s}$, respectively, then the projection distribution of $x^{-i}$ in this generated game is the same between $x_{s}^{-i}$ and $x_{s+1}^{-i}$. From the definition of $g_{x_{s}^{-i}}$ it follows $\hat{u}\left(x_{s}^{-i}\right) \geq \hat{u}\left(x_{s+1}^{-i}\right)$. We repeat the process above and obtain $g_{x_{s+2}^{-i}}$ and so on.

Because $\Gamma$ has finitely many nodes and $\Gamma$ is generic, we will come to an $x_{l}^{-i}$ that can generate $g_{x_{l}^{-i}}$ the same as $g_{x_{l-1}^{-i}}$, and so the set $H_{l}$ defined in (A.6) is a singleton. Note that at every node not played by player $i$ in the only play $h_{l}$ in $H_{l}, x_{l}^{-1}$ requires the move along $h_{l}$. Therefore, $\hat{u}\left(x_{l}^{-i}\right)=v^{i}\left(h_{l}\right)>v^{i}(\bar{h})$.

\section{A.4 Notations, operations and preliminary results for Theorem 4.2}

Denote the set of decision nodes in a finite extensive-form game by $N_{d}(G)$ and let $r(\Gamma):=\left|N_{d}(\Gamma)\right|$ for the game $\Gamma$. We sometimes use the shorthand $r$ for $r(\Gamma)$.

Lemma A.4. Given a $\mu$-dynamic $\left(x_{t}\right)_{t}$ on the $k$-player game $\Gamma$ with $\mu<$ $1 /\left(2^{r+2}\right)$ and given a positive number $\epsilon<1 /\left(2^{r+1}\right)$, suppose there exist a play $h:=\left(n_{1}, \ldots, n_{|h|}\right)$ and a time $t_{0}$ such that $\rho_{t_{0}}(h) \geq 1-\epsilon$ and $\left.\dot{\rho}(h)\right|_{t_{0}} \geq 0$. Then $\left.\dot{x}\left(n_{j}, n_{j+1}\right)\right|_{t_{0}} \geq 0$ for all $j$ with $1 \leq j<|h|$. 
Proof. First, note that when $r=1$, i.e., $\Gamma$ contains only one non-terminal node, the result is trivial.

We prove by contradiction for the case where $r>1$. Suppose the desired conclusion is not true. Then, at any $t$ with

$$
\rho_{t}(h) \geq 1-\epsilon>1-1 / 2^{r+1},
$$

there exists at least one $n_{j}$ such that $\left.\dot{x}\left(n_{j}, n_{j+1}\right)\right|_{t}<0$.

Without loss of generality, we assume that $\lambda\left(n_{i}\right) \neq \lambda\left(n_{l}\right)$ for all $i \neq l$; i.e., each non-terminal node in $h$ is played by a different player. It follows from $\rho_{t}(h)>1-1 / 2^{r+1}$ that $x_{t}\left(n_{j}, n_{j+1}\right)>1-1 / 2^{r+1}$ for all $j$ with $1 \leq j<|h|$. From

$$
\left(\prod_{j=1}^{|h|-1} x\left(n_{j}, n_{j}+1\right)\right)^{\prime}=\sum_{l=1}^{|h|-1}\left(\dot{x}\left(n_{l}, n_{l+1}\right) \prod_{j \neq l} x\left(n_{j}, n_{j+1}\right)\right)
$$

and the definition of $\mu$-dynamic, we may infer that

$$
\begin{aligned}
& \left.\dot{\rho}(h)\right|_{t} \\
= & \left.\left(\prod_{j=1}^{|h|-1} x\left(n_{j}, n_{j}+1\right)\right)^{\prime}\right|_{t} \\
\leq & (\epsilon+\mu)(|h|-2)-(1-\epsilon-\mu)^{|h|-1} \\
\leq & \left(\frac{1}{2^{r+1}}+\mu\right)(r-1)-\left(1-\frac{1}{2^{r+1}}-\mu\right)^{r} .
\end{aligned}
$$

Since $\mu<1 /\left(2^{r+2}\right)$,

$$
\left(\frac{1}{2^{r+1}}+\mu\right)(r-1) \leq \frac{5}{16}
$$

and

$$
\left(1-\frac{1}{2^{r+1}}-\mu\right)^{r} \geq\left.\left(1-\frac{5}{2^{r+2}}\right)^{r}\right|_{r=2}=\frac{121}{256}
$$

for all $r$ with $r>1$.

From (A.10), (A.11), and (A.12) it follows that $\left.\dot{\rho}(h)\right|_{t}<0$, a contradiction.

Recall that the set of plays is denoted by $H$. Given $\epsilon>0$ and a dynamic process $\left(x_{t}\right)_{t \geq 0}$, we denote

$$
S(\epsilon):=\left\{t: \rho_{t}(h)>1-\epsilon \text { and }\left.\dot{\rho}(h)\right|_{t}>0 \text { for some } h \in H\right\}
$$


and

$$
W(\epsilon):=\left\{t: \rho_{t}(h)>1-\epsilon \text { for some } h \in H\right\} .
$$

From Lemmata A.4 and 3.7, and replacing (3.16) by

$$
(r+1) \epsilon<\bar{\epsilon}
$$

(this adjustment is for an $\epsilon$-dynamic; see (3.17) and (3.18)), we obtain the following corollary.

Corollary A.5. Given the game $\Gamma$, we take any positive $\epsilon<1 /\left(2^{r+1}\right)$. For a $\mu$-dynamic $\left(x_{t}\right)_{t \geq 0}$ on $\Gamma$ with $\mu<\epsilon / 2$ and with the property (A.14), it follows that

$$
x_{t} \in S(\epsilon) \Rightarrow x_{t} \in N E[\epsilon] .
$$

We obtain the following theorem.

Theorem A.6. Given the game $\Gamma$, suppose there exists a positive number $\epsilon$ with the following properties:

1. $\epsilon<1 /\left(2^{r+1}\right)$;

2. (A.14) holds;

3.

$$
\frac{r \epsilon}{(1-\epsilon)^{r}-(r-1) \epsilon}<\frac{2 r \epsilon}{1-2 r \epsilon} .
$$

Given a positive number $T^{\prime}$, if an $\epsilon / 2$-dynamic satisfies

$$
\frac{1}{T} \int_{0}^{T} \mathbb{1}_{W(\epsilon)} \mathrm{d} t \geq 1-\epsilon
$$

for all $T>T^{\prime}$, then

$$
\frac{1}{T} \int_{0}^{T} \mathbb{1}_{\left\{t: x_{t} \in N E[\epsilon]\right\}} \mathrm{d} t \geq 1-(2 r+1) \epsilon
$$

for all $T>T^{\prime}$.

Proof. For an $\epsilon / 2$-dynamic on $\Gamma$, suppose that there exists a time $t_{1}$ and a play $h:=\left(n_{1}, \ldots, n_{|h|}\right)$ with $\rho_{t_{1}}(h) \geq 1-\epsilon$ and $\left.\dot{\rho}(h)\right|_{t_{1}}>0$. Then, by Lemma A.4, $\left.\dot{x}\left(n_{j}, n_{j+1}\right)\right|_{t_{1}} \geq 0$ for all $j$ with $1 \leq j<|h|$. Denote

$$
t_{2}:=\min \left\{t>t_{1}: \rho_{t}(h)=\rho_{t_{1}}(h)\right\} \text {. }
$$


We know that, when $\rho_{t}(h) \geq 1-\epsilon$ at any $t, x_{t}\left(n_{j}, n_{j+1}\right) \geq 1-\epsilon$ for all $j<|h|$. From the definition of an approximate best-response dynamic and (A.9), we know that when $\left.\dot{\rho}(h)\right|_{t} \geq 0,\left.\dot{\rho}(h)\right|_{t}<r \epsilon$; when $\left.\dot{\rho}(h)\right|_{t}<0,\left.\dot{\rho}(h)\right|_{t}<$ $-(1-\epsilon)^{r}+(r-1) \epsilon$. It follows from(A.17) and (A.15) that if $t_{2}$ is finite, then

$$
\int_{t_{1}}^{t_{2}} \mathbb{1}_{\left\{t:\left.\dot{\rho}(h)\right|_{t}<0\right\}} \mathrm{d} t<(2 r \epsilon)\left(t_{2}-t_{1}\right) .
$$

We then apply Corollary A.5 to complete the proof.

Tree operations: Recall that $N_{d}(G)$ is the set of decision nodes in the game $G$. Given a generic extensive-form game $G$ of perfect information with $\left|N_{d}(G)\right|=r(G)>1$, define the set of pre-terminal nodes in $G$ as

$$
L(G):=\left\{n \in N_{d}(G): \Psi^{-1}(n) \subseteq N_{t}(G)\right\} ;
$$

i.e., $L(G)$ is the subset of decision nodes whose successors are all terminal nodes. We take a node $\tilde{n} \in L(G)$ and introduce below two operations on game $G$.

1. Given the node $\tilde{n}$, we denote the last node played by player $\lambda(\tilde{n})$ before $\tilde{n}$ by $\hat{n}$. Then

$$
\Psi(\tilde{n}) \cap \Lambda^{\lambda(\tilde{n})}=\{\hat{n}\} \cup\left(\Psi(\hat{n}) \cap \Lambda^{\lambda(\tilde{n})}\right) .
$$

(If such an $\hat{n}$ does not exist, then we will not implement this operation on $G$ in the proof.) We then denote the node $n^{\prime}$ to be the immediate successor node of $\hat{n}$ in the play to $\tilde{n}$, i.e., $n^{\prime} \in \psi^{-1}(\hat{n}) \cap(\Psi(\tilde{n}) \cup\{\tilde{n}\})$. We define game $\tilde{G}$ to be the game with the edge $\left(\hat{n}, n^{\prime}\right)$ together with the subgame $G_{n^{\prime}}$ removed from $G$. Therefore $\left|N_{d}(\tilde{G})\right| \leq r(G)-1$.

Given the game $\tilde{G}$, for each player $i$, we derive the set $\tilde{B}^{i}$ of pure quasistrategies and the set $\tilde{X}^{i}$ in $\tilde{G}$ from the original game $G$ as follows. For each player $i$, we define a surjective function $q^{i}: B^{i} \rightarrow \tilde{B}^{i}$ such that, for every $b^{i}$ in $B^{i}, q^{i}\left(b^{i}\right)$ and $b^{i}$ have the same sequence of moves in $\tilde{G}$, i.e.,

$$
\tilde{b}^{i}(n)=b^{i}(n) \forall n \in N_{d}(\tilde{G}) \cap \bar{N}_{b^{i}}
$$

when $\tilde{b}^{i}=q^{i}\left(b^{i}\right)$. (Recall the definition of $\bar{N}_{b^{i}}$ for a pure quasi-strategy $b^{i}$ in the second paragraph in Section 3.1.) We may sometimes drop the superscript $i$ in $q^{i}$. We define $\tilde{X}^{i}$ as in (2.2). We may also view each $\tilde{b}^{i}$ in $\tilde{B}^{i}$ as a class of pure quasi-strategies in $\Gamma$, and hence define

$$
x^{i}\left(\tilde{b}^{i}\right):=\sum_{b^{i}: q\left(b^{i}\right)=\tilde{b}^{i}} x^{i}\left(b^{i}\right) .
$$


2. Denote $\psi(\tilde{n})$ by $\bar{n}$. In $\psi^{-1}(\tilde{n})$, denote by $\tilde{n}^{\prime}$ the terminal node with the maximum payoff to the player who moves at node $\tilde{n}$, i.e.,

$$
v^{\lambda(\tilde{n})}\left(\tilde{n}^{\prime}\right)=\max _{n \in \psi^{-1}(\tilde{n})} v^{\lambda(\tilde{n})}(n) .
$$

We define a new game $\bar{G}$ with $\left|N_{d}(\bar{G})\right|=r(G)-1$ by the following two steps.

(a) Remove subgame $G_{\tilde{n}}$ and the edge $(\bar{n}, \tilde{n})$. We arbitrarily index all remaining non-terminal nodes as $n^{1}, n^{2}, \ldots, n^{|r(G)-1|}$. For each node $n^{j}$, we add two terminal nodes $n_{1}^{j}$ and $n_{2}^{j}$ to $n^{j}$ with the property that

$$
v^{i}\left(n_{1}^{j}\right)=v_{\left(\left|N_{t}(G)\right|\right)}^{i}-j
$$

for all players $i ; v^{i}\left(n_{2}^{j}\right)=v_{(1)}^{i}+j$ for all players $i \neq \lambda\left(n^{j}\right)$ and $v^{\lambda\left(n^{j}\right)}\left(n_{2}^{j}\right)=v_{\left(\left|N_{t}(G)\right|\right)}^{\lambda\left(n^{j}\right)}-r(G)-j$. (Recall the definition of $v_{(l)}^{i}$ in the game $G$ at the start of Section 3.1.)

(b) Add a new terminal node $\tilde{n}$ to $\bar{n}$ with $\mathbf{v}_{\tilde{n}}=\mathbf{v}_{\tilde{n}^{\prime}}$, where $\tilde{n}^{\prime}$ is the terminal node denoted in (A.21) in $G$.

We can see that the generated game $\bar{G}$ is a generic game.

\section{A.5 Proof of Theorem 4.2}

We prove Theorem 4.2 by induction on the number of non-terminal nodes.

Induction hypothesis: Given a natural number $\eta \geq 1$ and any finite generic extensive-form game $G$ of perfect information with $r(G) \leq \eta$ and without chance nodes, for every $\epsilon>0$ there exist a number $\mu_{G}(\epsilon)$ with $0<\mu_{G}(\epsilon)<\epsilon$ and a number $T_{G}(\epsilon)>0$ such that for every interior $\mu_{G}(\epsilon)$ dynamic $\left(x_{t}\right)_{t}$ on $G$ with any initial state $x_{0}$

$$
\frac{1}{T} \int_{0}^{T} \mathbb{1}_{\left\{t: x_{t} \in N E[\epsilon]\right\}} \mathrm{d} t \geq 1-\epsilon
$$

for all $T \geq T_{G}(\epsilon)$. In the proof, we also apply a weaker version with (A.23) replaced by

$$
\frac{1}{T} \int_{0}^{T} \mathbb{1}_{W(\epsilon)} \mathrm{d} t \geq 1-\epsilon .
$$

(Recall $W(\epsilon)$ in (A.13).) Note that (A.23) trivially holds when $r(G)=1$.

For the game $\Gamma$ in Theorem 3.1, we let $r(\Gamma)=\eta+1>1$ and assume that the induction hypothesis above is true for all games $G$ with $r(G) \leq \eta$. We 
pick a node $\tilde{n}$ in $L(\Gamma)$ (defined in (A.18)) and suppose that player $i$ plays at node $\tilde{n}$.

Step I [when the dynamic in subgame $\Gamma_{\tilde{n}}$ can be ignored due to (A.25) below]

Recall the definition of $\bar{N}_{b^{i}}$ for a pure quasi-strategy $b^{i}$ in the second paragraph in Section 3.1.

Lemma A.7. Given a $\mu$-dynamic $\left(x_{t}\right)_{t \geq 0}$ on $\Gamma$ with $\mu<1 / 2$, suppose that

$$
\sum_{b^{i}: \tilde{n} \in \bar{N}_{b^{i}}} x_{t}^{i}\left(b^{i}\right)<\mu \forall t \geq 0
$$

For the game $\tilde{\Gamma}$ generated by Tree Operation 1 with respect to node $\tilde{n}$, there exists a $4 \mu$-dynamic $\left(\tilde{x}_{t}\right)_{t}$ on $\tilde{\Gamma}$ such that for all players $j \neq i, x_{t}^{j}\left(\tilde{b}^{j}\right)=\tilde{x}_{t}^{j}\left(\tilde{b}^{j}\right)$ for all $\tilde{b}^{j}$ in $\tilde{B}^{j}$ and for all $t \geq 0$ (recall $x_{t}^{j}\left(\tilde{b}^{j}\right)$ in (A.20)); for player $i$,

$$
\left|x_{t}^{i}\left(\tilde{b}^{i}\right)-\tilde{x}_{t}^{i}\left(\tilde{b}^{i}\right)\right| \leq 3 \mu
$$

for all $\tilde{b}^{i}$ in $\tilde{B}^{i}$ and for all $t \geq 0$. Moreover, for all players $j \neq i, \tilde{g}_{t}^{j}=q\left(g_{t}^{j}\right)$ for all $t \geq 0$, and, for player $i$, if $\tilde{n} \notin \bar{N}_{g_{t}^{i}}$ at time $t$, then $\tilde{g}_{t}^{i}=g_{t}^{i}$.

Proof. First, note that $\tilde{B}^{i}$ in $\tilde{\Gamma}$ is a subset of $B^{i}$ in $\Gamma$.

We define an ancillary process $\left(z_{t}^{i}\right)_{t \geq 0}$ on $G$ with the property that $z_{0}^{i}=x_{0}^{i}$ and $\dot{z}^{i}=\tilde{g}^{i}-z^{i}$ where the function $\tilde{g}_{t}^{i}:=g_{t}^{i}$ when $\tilde{n} \notin \bar{N}_{g_{t}^{i}}$ and $\tilde{g}_{t}^{i}$ is arbitrarily defined in $\tilde{B}^{i}$ when $\tilde{n} \in \bar{N}_{g_{t}^{i}}$. We show from the following observation that, in any such process $\left(z_{t}^{i}\right)_{t \geq 0}$, for any $t \geq 0$,

$$
\left|x_{t}^{i}\left(\tilde{b}^{i}\right)-z_{t}^{i}\left(\tilde{b}^{i}\right)\right| \leq \mu
$$

for all $\tilde{b}^{i} \in \tilde{B}^{i} \subseteq B^{i}$.

If for a period of time $\left[t_{1}, t_{2}\right]$ whenever $\tilde{g}_{t}^{i} \neq g_{t}^{i}$, we let $\tilde{g}_{t}^{i}$ be the same, say, that $\tilde{g}_{t}^{i}$ is some $\hat{b}^{i} \in \tilde{B}^{i}$ for all time $t$ in $\left[t_{1}, t_{2}\right]$ when $\tilde{g}_{t}^{i} \neq g_{t}^{i}$. If we further assume that $z_{t_{1}}\left(\hat{b}^{i}\right)=x_{t_{1}}\left(\hat{b}^{i}\right)$, then $z_{t_{1}}\left(\hat{b}^{i}\right)>x_{t_{1}}\left(\hat{b}^{i}\right)$. We may further infer that $z_{t}\left(\tilde{b}^{i}\right) \geq x_{t}\left(\tilde{b}^{i}\right)$ for all $\tilde{b}^{i} \in \tilde{B}^{i}$ and all $t \geq 0$. Hence we reach (A.26).

At $t=0$, for all players $j \neq i$, we let $\tilde{x}_{0}^{j}\left(\tilde{b}^{j}\right)=x_{0}^{j}\left(\tilde{b}^{j}\right)$ for all $\tilde{b}^{j}$ in $\tilde{B}^{j}$, and, for player $i$, we normalize $x_{0}^{i}$ in $\tilde{X}^{i}$ in such a way that

$$
\forall \tilde{b}^{i} \in \tilde{B}^{i}, \tilde{x}_{0}^{i}\left(\tilde{b}^{i}\right)=\frac{x_{0}^{i}\left(\tilde{b}^{i}\right)}{\sum_{\tilde{b}^{i} \in \tilde{B}^{i}} x_{0}^{i}\left(\tilde{b}^{i}\right)} .
$$

Then,

$$
\tilde{x}_{0}^{i}\left(\tilde{b}^{i}\right)<\frac{x_{0}^{i}\left(\tilde{b}^{i}\right)}{1-\mu}<(1+2 \mu) x_{0}^{i}\left(\tilde{b}^{i}\right) \forall \tilde{b}^{i} \in \tilde{B}^{i} .
$$


If in $\left(x_{t}\right)_{t \geq 0}, \tilde{n} \notin \bar{N}_{g_{t}^{i}}$ for all time $t \geq 0$, then the desired conclusion trivially holds. For the general case, consider the ancillary process $\left(z_{t}^{i}\right)_{t}$ above, and from (A.26) and (A.27) we can always set a $4 \mu$-dynamic $\left(\tilde{x}_{t}\right)_{t}$ such that for each player $j \neq i, \tilde{g}_{t}^{j}=q\left(g_{t}^{j}\right)$ for all $t>0$. Thus, we are allowed to set $\tilde{x}_{t}^{i}$ such that $\left|x_{t}^{i}\left(\tilde{b}^{i}\right)-\tilde{x}_{t}^{i}\left(\tilde{b}^{i}\right)\right| \leq 3 \mu$ for all $\tilde{b}^{i}$ in $\tilde{B}^{i}$ and for all $t>0$.

Lemma A.8. Given the game $\Gamma$ and $\epsilon>0$, there exists $\mu>0$ and $\bar{T}>0$ with the following property. For any interior $\mu$-dynamic in $\Gamma$ with the property (A.25) for some node $\tilde{n}$ in $L(\Gamma),(A .16)$ holds for all $T \geq \bar{T}$ in the $\mu$-dynamic.

Proof. Take $\epsilon_{\Gamma}$ that satisfies Conditions 1-3 in Theorem A.6. We denote $\min \left\{\epsilon / 4, \epsilon_{\Gamma}\right\}$ by $\epsilon_{1}$. Since $\left|N_{d}(\tilde{\Gamma})\right|<\left|N_{d}(\Gamma)\right|$, we apply the induction hypothesis on $\tilde{\Gamma}$ and obtain $\mu_{\tilde{\Gamma}}\left(\epsilon_{1}\right)<\epsilon_{1}$. Consider any positive $\mu \leq \mu_{\tilde{\Gamma}}\left(\epsilon_{1}\right) / 4$. By (A.24) in the induction hypothesis with respect to a $4 \mu$-dynamic in $\tilde{\Gamma}$ and Lemma A.7, we can find $T_{\tilde{\Gamma}}\left(\epsilon_{1}\right)$ such that in the interior $\mu$-dynamic in $\Gamma$

$$
\frac{1}{T} \int_{0}^{T} \mathbb{1}_{W\left(\epsilon_{1}+3 \mu\right)} \mathrm{d} t>1-\epsilon_{1}
$$

for all $T \geq T_{\tilde{\Gamma}}\left(\epsilon_{1}\right)$. Note $\epsilon_{1}+3 \mu<\epsilon$. Theorem A.6 completes the proof.

Step II [when $\tilde{n}$ can be viewed as a terminal node due to (A.28) and (A.29)]

Recall that node $\tilde{n}$ that we picked in $\Gamma$ is played by player $i$ and the game $\bar{\Gamma}$ is obtained by Tree Operation 2. (We can tell from the context whether $\tilde{n}$ refers to the decision node in $\Gamma$ or the terminal node in $\bar{\Gamma}$.) We define a function $\hat{q}: X \rightarrow \bar{X}$ with the property that if $\hat{q}(x)=\bar{x} \in \bar{X}$, then $x\left(n_{1}, n_{2}\right)=\bar{x}\left(n_{1}, n_{2}\right)$ for all edges $\left(n_{1}, n_{2}\right)$ in both $\Gamma$ and $\bar{\Gamma}$ when $x\left(n_{1}, n_{2}\right)$ is defined in $\Gamma$. (When we say $x\left(n_{1}, n_{2}\right)$ is defined, we mean that there exists a pure quasi-strategy $b^{\lambda\left(n_{1}\right)}$ such that $x^{\lambda\left(n_{1}\right)}\left(b^{\lambda\left(n_{1}\right)}\right)>0$ and $n_{1} \in \bar{N}_{b^{\lambda\left(n_{1}\right)}}$. See Section 3.1 for more details.) Thus, $\hat{q}(x)$ is the canonical projection of the mixed quasi-strategy profile $x$ in $\bar{\Gamma}$. Moreover, $\bar{x}\left(n, n_{1}\right)=\bar{x}\left(n, n_{2}\right)=0$, if $\bar{x}\left(n, n_{1}\right)$ and $\bar{x}\left(n, n_{2}\right)$ are defined, where $n_{1}$ and $n_{2}$ are the terminal nodes denoted in step (a) in Tree Operation 2.

Lemmata A.9, A.10, and A.11 show under what condition $B R^{l}(x) \subseteq$ $B R^{l}(\bar{x}[\mu])$ for a player $l \neq i$, if $\bar{x} \in \hat{q}(x)$.

Recall the constant $\delta$ defined for $\Gamma$ in (3.3). Denote the player who plays at node $\bar{n}$ by player $j$.

Lemma A.9. Given a mixed quasi-strategy profile $x$ in $\Gamma$ and a positive number $\mu$, we suppose that there exists a node $\tilde{n} \in L(\Gamma)$ with $\tilde{n}^{\prime}$ as defined in 
(A.21) and the property

$$
\begin{gathered}
0<\sum_{n \in \psi^{-1}(\tilde{n}) \backslash\left\{\tilde{n}^{\prime}\right\}} \sum_{b^{i}: \tilde{n} \in \bar{N}_{b^{i}}, b^{i}(\tilde{n})=n} x^{i}\left(b^{i}\right) \leq \frac{\mu^{4}}{4} \\
\frac{\mu^{2}}{\delta} \leq \sum_{b^{i}: \tilde{n} \in \bar{N}_{b^{i}}} x^{i}\left(b^{i}\right)<1 .
\end{gathered}
$$

We denote in game $\bar{\Gamma}$ (obtained by Tree Operation 2 with respect to node $\tilde{n}$ ) a mixed quasi-strategy profile $\bar{x}:=\hat{q}(x)$. Consider any $g^{j} \in B R^{j}\left(x\left[\mu^{4} / 4\right]\right)$ in $\Gamma$ and $\bar{g}^{j} \in B R^{j}(\bar{x})$ in $\bar{\Gamma}$. The following two conditions cannot hold at the same time.

1. In $\bar{\Gamma}$, there are two subplays $\left(n_{1}^{1}, n_{2}^{1}, \ldots, n_{k_{1}}^{1}\right)$ and $\left(n_{1}^{2}, n_{2}^{2}, \ldots, n_{k_{2}}^{2}\right)$ with $n_{k_{1}}^{1}=\tilde{n} \neq n_{k_{2}}^{2}, n_{k_{2}}^{2} \in N_{t}(\bar{\Gamma})$ and $n_{1}^{1}=n_{1}^{2}$. All decision nodes in these two subplays in $\bar{\Gamma}$ are played by player $j$. (Recall that player $j$ plays at node $\bar{n}$.)

2. For $i=1$ or 2, $n_{l}^{i} \in \bar{N}_{g^{j}}$ and $g^{j}\left(n_{l}^{i}\right)=n_{l+1}^{i}$ for all $l<k_{1} ; n_{l}^{3-i} \in \bar{N}_{\bar{g}^{j}}$ and $\bar{g}^{j}\left(n_{l}^{3-i}\right)=n_{l+1}^{3-i}$ for all $l<k_{3-i}$.

Proof. It follows from (A.28) and (A.29) that the local behaviour strategy of player $i$ at node $\tilde{n}$ in $\Gamma$ satisfies

$$
x^{\prime}\left(\tilde{n}, \tilde{n}^{\prime}\right) \geq \frac{\frac{\mu^{2}}{\delta}-\frac{\mu^{4}}{2}}{\frac{\mu^{2}}{\delta}}>1-\frac{\delta}{2} .
$$

for all $x^{\prime} \in x\left[\mu^{4} / 4\right]$. We complete the proof by the definition of $\delta$ in (3.3).

Recall that the decision node $\tilde{n}$ is played by player $i$ in $\Gamma$. Note that $\bar{B}^{l}=B^{l}$ for all $l \neq i$.

Lemma A.10. Given any $x$ in $X$ in $\Gamma$, we obtain $\bar{x}:=\hat{q}(x)$ in $\bar{\Gamma}$. For any player $l \neq i$, we consider a best reply $g^{l} \in B R^{l}(x)$ and $a \bar{g}^{l} \in B R^{l}(\bar{x})$ in two games $\Gamma$ and $\bar{\Gamma}$, respectively. If $g^{l} \neq \bar{g}^{l}$, then there is a node $n^{l}$ such that $n^{l} \in \bar{N}_{g^{l}} \cap \bar{N}_{\bar{g}^{l}}$, and, for all $n$ in $\Psi^{-1}\left(n^{l}\right) \cap \Lambda^{l}, n \notin \bar{N}_{g^{l}} \cap \bar{N}_{\bar{g}^{l}}$. For any $x$, we can always find a pair $\left(g^{l}, \bar{g}^{l}\right)$ such that, if $g^{l} \neq \bar{g}^{l}$, then there is at most one such node $n^{l}$ and

$$
\Psi^{-1}\left(n^{l}\right) \ni \tilde{n}
$$

in $\bar{\Gamma}$.

Remark: A node $n^{l}$ is where $g^{l}$ and $\bar{g}^{l}$ are deviating from each other. 
Proof. This follows from $\bar{x}=\hat{q}(x)$ and the fact that the projection distribution of $x$ in $\Gamma_{\bar{n}}$ is different from the projection of $\bar{x}$ in $\bar{\Gamma}_{\bar{n}}$.

Lemma A.11. We continue with the setting in Lemma A.10 and assume that only one node $n^{l}$ exists with property (A.30). We define a set $\hat{N}$ with the property $\hat{N} \subseteq\left(\Psi^{-1}\left(n^{l}\right) \cap \Lambda^{-l}\right)$, and for each $\hat{n} \in \hat{N}$ all nodes $n$ in $\Psi(\hat{n}) \cap$ $\Psi^{-1}\left(n^{l}\right)$ are in $\Lambda^{l}$.

If $\hat{N} \neq \emptyset$ and both (A.28) and (A.29) hold, then there exist $a \bar{z} \in \bar{x}\left[\mu^{2} / 4\right]$ and $a \bar{g}^{l}(\bar{z}) \in B R^{l}(\bar{z})$ such that $\bar{g}^{l}(\bar{z})=g^{l}$.

Remark: $\hat{N}$ is the subset in $\Psi^{-1}\left(n^{l}\right)$ that contains all first nodes not played by player $l$ after $n^{l}$. For any two nodes $n^{1}, n^{2} \in \hat{N}, n^{1} \notin \Psi\left(n^{2}\right) \cup$ $\Psi^{-1}\left(n^{2}\right)$.

Proof. First, note that one and only one of the following statements is true:

$$
g^{l}\left(n^{l}\right) \in \Psi(\tilde{n}) \cap\{\tilde{n}\} \text { and } \bar{g}^{l}\left(n^{l}\right) \notin \Psi(\tilde{n})
$$

or

$$
\bar{g}^{l}\left(n^{l}\right) \in \Psi(\tilde{n}) \cap\{\tilde{n}\} \text { and } g^{l}\left(n^{l}\right) \notin \Psi(\tilde{n}) .
$$

We first assume that (A.31) holds, and we generate $\bar{z} \in \bar{x}\left[\mu^{2} / 4\right]$ as follows. For the local behavior strategy at every node $n \in N_{d}(\bar{\Gamma}) \backslash \hat{N}$, we let $\bar{z}\left(n, n^{\prime}\right)=$ $\bar{x}\left(n, n^{\prime}\right)$ whenever $\bar{x}\left(n, n^{\prime}\right)$ is defined in $\bar{\Gamma}$.

If $\hat{N} \cap \Psi(\tilde{n}) \neq \emptyset$, then from the remark above there is only one node $\hat{n}$ in $\hat{N} \cap \Psi(\tilde{n})$, and we let $\bar{z}\left(\hat{n}, \hat{n}_{2}\right)=\mu^{2} / 4$, where $\hat{n}_{2}$ is defined in step (a) in Tree Operation $2 ; \bar{z}\left(\hat{n}, \hat{n}^{\prime}\right)=\left(1-\mu^{2} / 4\right) \bar{x}\left(\hat{n}, \hat{n}^{\prime}\right)$ for all nodes $\hat{n}^{\prime}$ in $\psi^{-1}(\hat{n}) \backslash\left\{\hat{n}_{2}\right\}$.

For every node $\hat{n}$ in $\hat{N} \backslash \Psi(\tilde{n})$, we let $\bar{z}\left(\hat{n}, \hat{n}_{1}\right)=\mu^{2} / 4$, where $\hat{n}_{1}$ is defined in step (a) in Tree Operation $2 ; \bar{z}\left(\hat{n}, \hat{n}^{\prime}\right)=\left(1-\mu^{2} / 4\right) \bar{x}\left(\hat{n}, \hat{n}^{\prime}\right)$ for all nodes $\hat{n}^{\prime}$ in $\psi^{-1}(\hat{n}) \backslash\left\{\hat{n}_{1}\right\}$.

If (A.32) holds, we apply a similar process to obtain $\bar{z}$ but with $\hat{n}_{1}$ and $\hat{n}_{2}$ swapped.

We can check that there exists $\bar{z}$ such that $\bar{z} \in \bar{x}\left[\mu^{4} / 4\right]$ and $\bar{g}^{l}(\bar{z})=g^{l}$.

Lemma A.12. Given a $\mu^{4} / 4$-dynamic $\left(x_{t}\right)_{t \geq 0}$ on $\Gamma$, we suppose that (A.28) and (A.29) hold at all $t \geq 0$. Then there exists a $\mu^{2} / 2$-dynamic $\left(\bar{x}_{t}\right)_{t \geq 0}$ in $\bar{\Gamma}$ (obtained by Tree Operation 2) with the property

$$
\bar{x}_{t}=\hat{q}\left(x_{t}\right) .
$$

at all $t \geq 0$. 
Proof. We define a $\mu^{2} / 2$-dynamic $\left(\bar{x}_{t}\right)_{t \geq 0}$ on $\bar{\Gamma}$ with the following propositions. Recall the step (a) in Tree Operation 2. For any decision node $n$ in $\bar{\Gamma}$, we denote $S_{n}^{1}:=\left\{b \in \bar{B}^{\lambda(n)}: n \in \bar{N}_{b}, b(n)=n_{1}\right\}$ and $S_{n}^{2}:=\left\{b \in \bar{B}^{\lambda(n)}\right.$ : $\left.n \in \bar{N}_{b}, b(n)=n_{2}\right\}$. We require that (A.33) holds at time $t=0$. Therefore, for each decision node $n$ in $\bar{\Gamma}$ and the associated two terminal nodes $n_{1}, n_{2} \in \psi^{-1}(n)$, it follows that

$$
\sum_{b \in S_{n}^{1}} \bar{x}_{0}^{\lambda(n)}(b)=\sum_{b \in S_{n}^{2}} \bar{x}_{0}^{\lambda(n)}(b)=0 .
$$

From the propositions of node $n_{1}$ and $n_{2}$ and the definition of $\mu$-dynamics, we know that

$$
\sum_{b \in S_{n}^{1}} \bar{x}_{t}^{\lambda(n)}(b)=\sum_{b \in S_{n}^{2}} \bar{x}_{t}^{\lambda(n)}(b)=0
$$

for all $t \geq 0$. We show below that there exists such $\mu^{2} / 2$-dynamic $\left(\bar{x}_{t}\right)_{t \geq 0}$ on $\bar{\Gamma}$ that satisfies (A.33) for all $t \geq 0$.

When $\bar{x}=\hat{q}\left(x_{t}\right)$, it is straightforward to see that $B R^{i}\left(x_{t}\left[\mu^{4} / 4\right]\right)$ in $\Gamma$ a subset of $B R^{i}\left(\bar{x}\left[\mu^{4} / 4\right]\right)$, where player $i$ moves at the node $\tilde{n}$ in $\Gamma$. Recall that player $j$ moves at node $\bar{n}$. For a player $l=j$ and with $\hat{N} \neq \emptyset$ (defined in Lemma A.11), or a player $l$ with $l \neq j$ and $l \neq i$, which induces $\hat{N} \neq \emptyset$, we can always adjust $\bar{x}\left(n, n_{1}\right)$ and $\bar{x}\left(n, n_{2}\right)$ in some local behavior strategies as shown in the proof of Lemma A.11, and make $B R^{l}\left(x_{t}\left[\mu^{4} / 4\right]\right)$ in $\Gamma$ a subset of $B R^{l}\left(\bar{x}\left[\mu^{2} / 2\right]\right)$ in $\bar{\Gamma}$. (The final adjustment consists of two parts: the adjustment shown in Lemma A.11 and imitation of $\bar{x}_{t}$ to the $x \in x_{t}\left[\mu^{4} / 4\right]$.)

Note that if player $l=j$ and $\hat{N}=\emptyset$, then we cannot adjust in that way to make $B R^{j}\left(x_{t}\left[\mu^{4} / 4\right]\right) \subseteq B R^{j}\left(\bar{x}\left[\mu^{2} / 2\right]\right)$, since player $j$ 's best response cannot include a move to her worst payoff. However, $\hat{N}=\emptyset$ leads to Condition 1 in Lemma A.9, and it follows that any $g_{t}^{j} \in B R^{j}\left(x_{t}\left[\mu^{4} / 4\right]\right)$ is also a best reply to some $\bar{x}^{\prime} \in \hat{q}\left(x_{t}\right)\left[\mu^{4} / 4\right]$ in $\bar{\Gamma}$. That completes the proof.

Lemma A.13. Given the game $\Gamma$ and $\epsilon>0$, there exist $\bar{\mu}$ with $0<\bar{\mu}<\epsilon$ and $\bar{T}>0$ with the following property. For any interior $\mu^{4} / 4$-dynamic $\left(x_{t}\right)_{t}$ in $\Gamma$ with $\mu<\bar{\mu}$ and the properties (A.28) and (A.29) at some node $\tilde{n} \in L(\Gamma)$ for all $t \geq 0$, (A.16) holds for all $T \geq \bar{T}$.

Proof. Take $\epsilon_{\Gamma}$ that satisfies Conditions 1-3 in Theorem A.6. We denote $\min \left\{\epsilon / 2, \epsilon_{\Gamma}\right\}$ by $\epsilon_{2}$. Since $\left|N_{d}(\tilde{\Gamma})\right|<\left|N_{d}(\Gamma)\right|$, we apply the induction hypothesis on $\bar{\Gamma}$ and obtain $\mu_{\bar{\Gamma}}\left(\epsilon_{2}\right)<\epsilon_{2}$. Take $\bar{\mu}:=\left(2 \mu_{\bar{\Gamma}}\left(\epsilon_{2}\right)\right)^{1 / 2}$, and consider any $\mu<\bar{\mu}$. Note that for the dynamic $\left(x_{t}\right)_{t}$ with the properties (A.28) and (A.29) and the associated dynamic $\left(\bar{x}_{t}\right)_{t}$ studied in Lemma A.12, if $\bar{\rho}_{t}(\bar{h})=p$ in $\left(\bar{x}_{t}\right)_{t}$ for the play $\bar{h}$ up to the terminal node $\tilde{n}$ in $\bar{\Gamma}$, then, for $\left(x_{t}\right)_{t}, \rho_{t}(h) \geq p\left(1-\mu^{2} / 4\right)$ for the play $h=\bar{h} \cup\left\{\tilde{n}^{\prime}\right\}$ (in $\Gamma$ ). By (A.24) in 
the induction hypothesis with respect to a $\mu^{2} / 2$-dynamic in $\bar{\Gamma}$ and Lemma A.12, we can find $T_{\bar{\Gamma}}\left(\epsilon_{2}\right)$ such that for any interior $\mu^{2} / 4$-dynamic in $\Gamma$

$$
\frac{1}{T} \int_{0}^{T} \mathbb{1}_{W\left(\epsilon_{2}+\mu^{2} / 4\right)} \mathrm{d} t>1-\epsilon_{2}
$$

for all $T>T_{\bar{\Gamma}}\left(\epsilon_{2}\right)$. From $\mu^{2} / 4<\mu_{\bar{\Gamma}}\left(\epsilon_{2}\right) / 2<\epsilon_{2} / 2<\epsilon / 4$, it follows that

$$
\frac{1}{T} \int_{0}^{T} \mathbb{1}_{W(\epsilon)} \mathrm{d} t>1-\epsilon / 2 .
$$

Theorem A.6 completes the proof.

Step 3: [Find a small $\mu$.]

We first give a corollary from Lemmata A.8 and A.13.

Corollary A.14. Given the game $\Gamma$ and $\epsilon>0$, there exists $\hat{\mu}$ with $0<\hat{\mu}<\epsilon$ and $\hat{T}>0$ with the following property. For any interior $\mu^{4} / 4$-dynamic $\left(x_{t}\right)_{t}$ in $\Gamma$ with $\mu<\hat{\mu}$, any initial state $x_{0}$, and either of the two conditions below at some node $\tilde{n} \in L(\Gamma)$ for all $t \geq 0$,

1. (A.25)

2. the conjunction of (A.28) and (A.29),

it follows that

$$
\int_{0}^{T} \mathbb{1}_{\left\{t: x_{t} \in N E[\epsilon]\right\}} \mathrm{d} t \geq T(1-\epsilon)
$$

for all $T \geq \hat{T}$.

Proof of Theorem 4.2. Recall $\tilde{n} \in L(\Gamma)$, and two associated games $\tilde{\Gamma}$ and $\bar{\Gamma}$ defined by Tree Operation 1 and Tree Operation 2, respectively. Recall that in $\Gamma$ node $\tilde{n}$ is played by player $i$. We first note that for any interior approximate best-response dynamic $\left(x_{t}\right)_{t \geq 0}$ on $\Gamma$, for any possible best reply $g_{t}^{i}$ of player $i$ at any time $t$, if $\tilde{n} \in \bar{N}_{g_{t}^{i}}$, then $g_{t}^{i}(\tilde{n})$ must be $\tilde{n}^{\prime}$. Therefore, for any $c>0$, there exists a time $\bar{t}$ such that

$$
\forall t \geq \bar{t}, \quad \sum_{n \in \psi^{-1}(\tilde{n}) \backslash\left\{\tilde{n}^{\prime}\right\}} \sum_{b^{i}: \tilde{n} \in \bar{N}_{b^{i}}, b^{i}(\tilde{n})=n} x_{t}^{i}\left(b^{i}\right) \leq c
$$

for all approximate best-response dynamics $\left(x_{t}\right)_{t \geq 0}$.

We take $\mu$ under the following constraints: 
1.

$\mu<\delta / 2$

2 .

$\hat{T}<\ln \delta-\ln (2 \mu)$

where $\hat{T}$ is defined in Corollary A.14;

3. $\mu<\hat{\mu}$ in Corollary A.14.

We consider any interior $\mu^{4} / 4$-dynamic $\left(x_{t}\right)_{t}$ with the $\mu$ taken under the above constraints, and then transform the original dynamic to a dynamic $\left(z_{t}\right)_{t}$ with $z_{t}=x_{t+\bar{t}}$ where (A.35) holds with $c$ replaced by $\mu^{4} / 4$ for all $t \geq \bar{t}$. We define a sequence of times $\left(t_{k}\right)_{k \geq 0}$ with $t_{0}=0$ such that for any $t \geq 0$

$$
t_{2 k+1}=\min \left\{t \geq t_{2 k}: \sum_{b^{i}: \tilde{n} \in \bar{N}_{b^{i}}} z_{t}^{i}\left(b^{i}\right) \geq \mu\right\}
$$

and

$$
t_{2 k+2}=\min \left\{t \geq t_{2 k+1}: \sum_{b^{i}: \tilde{n} \in \bar{N}_{b^{i}}} z_{t}^{i}\left(b^{i}\right)<\frac{\mu^{2}}{\delta}\right\} .
$$

Note that it is possible that there exists a natural number $l$ such that $t_{k}=$ $+\infty$ for all $k \geq l$. During $\left[t_{2 k+1}, t_{2 k+2}\right)$ for any $k \geq 0$, we can apply conditions (A.28) and (A.29) of Corollary A.14. From (A.36), it follows that $\mu / 2>\mu^{2} / \delta$. From (3.8), we may further infer that

$$
\begin{aligned}
& t_{2 k+2}-t_{2 k+1}>\min \left\{t>t_{2 k+1}: \sum_{b^{i}: \tilde{n} \in \bar{N}_{b^{i}}, b^{i}(\tilde{n})=\tilde{n}^{\prime}} z_{t}^{i}\left(b^{i}\right)=\frac{\mu}{2}\right\} \\
&-\min \left\{t>t_{2 k+1}: \sum_{b^{i}: \tilde{n} \in \bar{N}_{b^{i}}, b^{i}(\tilde{n})=\tilde{n}^{\prime}} z_{t}^{i}\left(b^{i}\right)=\frac{\mu^{2}}{\delta}\right\} \\
&> \ln \frac{\delta}{\mu^{2}}-\ln \frac{2}{\mu} \\
&= \ln \delta-\ln (2 \mu) .
\end{aligned}
$$

From the definition of $t_{2 k+1}$, it follows that, for any $t$ with $t_{2 k} \leq t<t_{2 k+1}$,

$$
\sum_{b^{i}: \tilde{n} \in \bar{N}_{b^{i}}} z_{t}^{i}\left(b^{i}\right)<\mu
$$


We can then apply condition (A.25) of Corollary A.14 for any $\left[t_{2 k}, t_{2 k+1}\right.$ ) with $k \geq 0$. Recall (A.37), and we may then infer from Corollary A.14 that, during $\left[t_{2 k+1}, t_{2 k+2}\right)$ for any $k \geq 0$,

$$
\frac{1}{t_{2 k+2}-t_{2 k+1}} \int_{2 k+1}^{2 k+2} \mathbb{1}_{\left\{t: z_{t} \in N E[\epsilon]\right\}} \mathrm{d} t>1-\epsilon .
$$

Moreover, by Corollary A.14, we can see from (A.38) and (A.37) that during $\left(t_{2 k}, t_{2 k+3}\right)$ for any $k \geq 0$,

$$
\frac{1}{t_{2 k+3}-t_{2 k}} \int_{2 k}^{2 k+3} \mathbb{1}_{\left\{t: z_{t} \notin N E[\epsilon]\right\}} \mathrm{d} t<\frac{3 \hat{T} \epsilon}{t_{2 k+2}-t_{2 k+1}}<3 \epsilon .
$$

Therefore, in the original $\mu^{4} / 4$-dynamic $\left(x_{t}\right)_{t}$,

$$
\frac{1}{T} \int_{\bar{t}}^{\bar{t}+T} \mathbb{1}_{\left\{t: x_{t} \in N E[\epsilon]\right\}} \mathrm{d} t \geq 1-3 \epsilon
$$

for all $T \geq 2 \hat{T}$. We finally let $\mu(4 \epsilon):=\mu^{2} / 4$ and $T(4 \epsilon):=\bar{t} / \epsilon+2 \hat{T}$. It follows that for any $\mu(4 \epsilon)$-dynamic on $\Gamma$ with any initial state

$$
\frac{1}{T} \int_{0}^{T} \mathbb{1}_{\left\{t: x_{t} \in N E[4 \epsilon]\right\}} \mathrm{d} t \geq 1-4 \epsilon
$$

for all $T \geq T(4 \epsilon)$. We have thus shown that the induction hypothesis can also be applied to game $\Gamma$, and indeed to all generic extensive-form games of perfect information with $\eta+1$ decision nodes (without chance nodes).

\section{A.6 Proofs in Section 5}

Proof of Theorem 5.1. We prove it by induction on the number of nonterminal nodes, with the following induction hypothesis. Denote by $\left|N_{d}(G)\right|$ the number of non-terminal nodes in the game $G$.

Induction hypothesis: Given a natural number $\eta \geq 1$ and any finite generic extensive-form game $G$ of perfect information with $\left|N_{d}(G)\right|=\eta$, for every $\epsilon>0$ there exist a number $\mu_{G}(\epsilon)$ with $0<\mu_{G}(\epsilon)<\epsilon$ and a number $T_{G}(\epsilon)>0$ such that for every interior $\mu_{G}(\epsilon)$-dynamic $\left(x_{t}\right)_{t}$ on $G$ with any initial state $x_{0}$

$$
x_{t} \in B I E[\epsilon]
$$

for all $t \geq T_{G}(\epsilon)$. Note that this assumption trivially holds when $\left|N_{d}(G)\right|=1$.

We prove Theorem 5.1 for the game $\Gamma$ with $\left|N_{d}(\Gamma)\right|=\eta+1>1$ under the assumption that the induction hypothesis above is true for all games $G$ 
with $\left|N_{d}(G)\right|=\eta$. We pick a node $\tilde{n}$ in $L(\Gamma)$. (Recall the definition of $L(\Gamma)$ in (A.18).) If $\tilde{n}$ is a chance node, then by the generic assumption, the desired result trivially holds. Given $\mu>0$, we can see that there exists a time $t_{0}$ such that, for any interior $\mu$-dynamic $\left(x_{t}\right)_{t}$, both (A.28) and (A.29) hold for all times $t \geq t_{0}$.

We obtain the game $\bar{\Gamma}$ by Tree Operation 2 in Section A.4, and a $\mu_{\bar{\Gamma}}(\epsilon / 2)<$ $\epsilon / 2$. Take $\bar{\mu}:=\left(2 \mu_{\bar{\Gamma}}(\epsilon / 2)\right)^{1 / 2}$, and consider any $\mu<\bar{\mu}$. Note that the backward-induction equilibrium path in $\bar{\Gamma}$ is included in the backward-induction equilibrium path in $\Gamma$. By (A.40) in the induction hypothesis with respect to a $\mu^{2} / 2$-dynamic in $\bar{\Gamma}$ and Lemma A.13, we can find $T_{\bar{\Gamma}}(\epsilon / 2)$ such that for any $\mu^{4} / 4$-dynamic $\left(x_{t}\right)_{t}$ in $\Gamma$

$$
x_{t} \in B I E\left[\frac{\epsilon}{2}+\frac{\mu^{2}}{4}\right]
$$

for all $t>T_{\bar{\Gamma}}(\epsilon / 2)+t_{0}$. Note $\mu^{2} / 4<\mu_{\bar{\Gamma}}(\epsilon / 2) / 2<\epsilon / 4$, and we have completed the proof.

Proof of Theorem 5.2. We prove it by induction on the number of nonterminal nodes, with the following induction hypothesis. Denote by $\left|N_{d}(G)\right|$ the number of non-terminal nodes in the game $G$.

Induction hypothesis: Given a natural number $\eta \geq 1$ and any finite generic extensive-form game $G$ of perfect information with $\left|N_{d}(G)\right|=\eta$ and $\epsilon^{\prime}>0$, there exists a number $T_{G}\left(\epsilon^{\prime}\right)>0$ such that for any initial state $x_{0}$ and any $\mu$ with $0<\mu<\epsilon^{\prime}$, there exists a $\mu$-dynamic $\left(x_{t}\right)_{t}$ on $G$ with the property

$$
x_{t} \in B I E\left[\epsilon^{\prime}\right]
$$

for all $t>T_{G}\left(\epsilon^{\prime}\right)$. Note that the above assumption trivially holds when $\left|N_{d}(G)\right|=1$.

We prove Theorem 5.2 for the game $\Gamma$ with $\left|N_{d}(\Gamma)\right|=\eta+1>1$ under the assumption that the induction hypothesis above is true for all games $G$ with $\left|N_{d}(G)\right|=\eta$. We take the last non-terminal node in a backwardinduction equilibrium path as $\tilde{n}$, and so $\tilde{n} \in L(\Gamma) \cap h_{B C}$, where $h_{B C}$ denotes a backward-induction equilibrium path. If $\tilde{n}$ is a chance node, then by the generic assumption, the desired result trivially holds.

Given $\mu>0$, we can see that there exists a time $t_{0}$ such that, for any $\mu / 2$-dynamic $\left(x_{t}\right)_{t},\left(\right.$ A.28) holds for all $t \geq t_{0}$.

We apply the function $\hat{q}$ defined at the start of Step 2 in the proof of Theorem 4.2 , and let $\bar{x}_{0}=q\left(x_{t_{0}}\right)$. We obtain the game $\bar{\Gamma}$ by Tree Operation 2 with respect to $\tilde{n}$. We apply the induction hypothesis on $\bar{\Gamma}$ with respect to 
$\epsilon^{\prime}=\epsilon / 2$ and obtain $T_{\bar{\Gamma}}(\epsilon / 2)$ and a $\mu / 2$-dynamic $\left(\bar{x}_{t}\right)_{t}$ with initial state $\bar{x}_{0}$ and property (A.41). For all players $i$ and all $t \geq t_{0}$, we can simply let $g_{t+t_{0}}^{i}=\bar{g}_{t}^{i}$, and we find $x_{t+t_{0}} \in \bar{x}_{t}\left[\mu^{4} / 4\right]$ and $g_{t+t_{0}}^{i} \in B R^{i}(x[\mu])$. Therefore, $\left(x_{t}\right)_{t \geq t_{0}}$ is a $\mu$-dynamic. From (A.41), we then infer that

$$
x_{t} \in B I E[\epsilon]
$$

for all $t>t_{0}+T_{\bar{\Gamma}}(\epsilon / 2)$. 


\section{References}

Aubin, J., and A. Cellina (1984): Differential Inclusions. Berlin: Springer.

Benaim, M., and J. W. Weibull (2003): "Deterministic Approximation of Stochastic Evolution in Games," Econometrica, 71, 873-903.

Cressman, R. (2003): Evolutionary Dynamics and Extensive Form Games. Cambridge (MA): MIT Press.

Cressman, R., and K.H. Schlag (1998): "The Dynamic (in)Stability of Backward Induction," Journal of Economic Theory, 83, 260-285.

Gilboa, Y., and A. Matsui (1991): "Social Stability and Equilibrium," Econometrica, 59, 859-867.

Gorodeisky, Z. (2006): "Evolutionary Stability for Large Populations and Backward Induction," Mathematics of Operations Research, 31, 369-380.

Hart, S. (1992): Games in Extensive and Strategic Forms. In R.J. Aumann and S. Hart, editors, Handbook of Game Theory with Economic Applications, Elsevier, Edition 1, Volume 1, North Holland.

Hart, S. (2002): "Evolutionary Dynamics and Backward Induction," Games and Economic Behavior, 41, 227-264.

Hart, S. (2005): “Adaptive Heuristics," Econometrica, 73 (5), 1401-1430.

Hart, S., and A. Mas-Colell (2003): "Regret-Based Continuous-Time Dynamics," Games and Economic Behavior, 45, 375-394.

Hart, S., and A. Mas-Colell (2005): "Uncoupled Dynamics do not Lead to Nash Equilibrium," American Economic Review, 93, 1830-1836.

Hofbauer, J. (1995): "Stability for the Best Response Dynamics," mimeo, University of Vienna.

Hofbauer, J., and W. H. Sandholm (2002): "On the Global Convergence of Stochastic Fictitious Play," Econometrica, 70, 2265-2294.

Hofbauer, J., and K. Sigmund (1998): Evolutionary Games and Population Dynamics. Cambridge University Press.

Kandori, M., G. Mailath, and R. Rob (1993): "Learning, Mutation, and Long-Run Equilibrium in Games," Econometrica, 61, 29-56. 
Kandori, M., and R. Rob (1995): "Evolution of Equilibria in the Long Run: A General Theory and Applications," Journal of Economics Theory, 65, 383-414.

Kreps, D., and R. Wilson (1982): "Sequential Equilibria," Econometrica, 50, 863-894.

Kuhn, H. W. (1953): Extensive Games and the Problem of Information. In H. W. Kuhn and A. W. Tucker, editors, Contributions to the Theory of Games II. Annals of Mathematics Studies, Vol. 28, Princeton University Press.

Matsui, A. (1998): "Social Stability and Equilibrium," CMS-DMS No. 819, Northwestern University.

Nash, J. F. (1950): Non-Cooperative Games, PhD Dissertation at Princeton University.

Nöldeke, G., and L. Samuelson (1993): "An Evolutionary Analysis of Backward and Forward Induction," Games and Economic Behavior, 5, 425-454.

Ritzberger, K. (2002): Foundations of Non-Cooperative Game Theory. Oxford University Press.

Ritzberger, K., and J. Weibull (1995): "Evolutionary Selection in NormalForm Games," Econometrica, 63, 1371-1399.

Sandholm, W. H., Population Games and Evolutionary Dynamics. MIT Press.

Xu, Z. (2013): "Stochastic Stability in Finite Extensive-Form Games of Perfect Information," SSE/EFI Working Paper Series in Economics and Finance, No. 743.

Young, H. P. (2009): "Learning by Trial and Error," Games and Economic Behavior, 65, 626-643. 